\title{
A framework for topology optimization of inertial microfluidic particle manipulators
}

\section{Andreasen, Casper Schousboe}

Published in:

Structural and Multidisciplinary Optimization

Link to article, DOI:

$10.1007 / \mathrm{s} 00158-019-02483-5$

Publication date:

2020

Document Version

Peer reviewed version

Link back to DTU Orbit

Citation $(A P A)$ :

Andreasen, C. S. (2020). A framework for topology optimization of inertial microfluidic particle manipulators. Structural and Multidisciplinary Optimization, 2481-2499. https://doi.org/10.1007/s00158-019-02483-5

\section{General rights}

Copyright and moral rights for the publications made accessible in the public portal are retained by the authors and/or other copyright owners and it is a condition of accessing publications that users recognise and abide by the legal requirements associated with these rights.

- Users may download and print one copy of any publication from the public portal for the purpose of private study or research.

- You may not further distribute the material or use it for any profit-making activity or commercial gain

- You may freely distribute the URL identifying the publication in the public portal

If you believe that this document breaches copyright please contact us providing details, and we will remove access to the work immediately and investigate your claim. 


\title{
A Framework for Topology Optimization of Inertial Microfluidic Particle Manipulators
}

\author{
Casper Schousboe Andreasen
}

Received: date / Accepted: date

\begin{abstract}
The design of components for inertial microfluidic focusing and separation is primarily designed from basic geometric primitives. This paper presents a topology optimization methodology to the design synthesis of microfluidic particle manipulators. The flow is modeled by the Navier-Stokes equations in an Eulerian frame while the particle transport is modeled as a Lagrangian particle model. The model assumes that the particles are small and the suspension is dilute such that both the particle influence on the fluid motion and collisions between particles can be neglected. Two manipulator design problems are studied - one that makes particles follow a predefined trajectory, and another where particles are focused at the outlet utilizing the inertia of the particles. The latter is extracted and post analyzed using a commercial software COMSOL verifying the method's ability to synthesize inertial microfluidic components.
\end{abstract}

Keywords Particle transport - microfluidics - Topology Optimization · Particle tracking

\section{Introduction}

Particle control and the ability to focus or sort particles in microfluidic components is very useful in lab-on-achip size detectors and manipulators, portable point-ofcare diagnostic devices and in micro-total-analysis systems ( $\mu$ TAS). These devices may be applied in biomedical research for the improved detection and analysis

Casper Schousboe Andreasen

Department of Mechanical Engineering, Section for Solid Mechanics, Technical University of Denmark, Nils Koppels Allé, Building 404, DK-2800 Kgs. Lyngby

Tel.: +45 45254262

E-mail: csan@mek.dtu.dk of cells, e.g. circulating tumor cells (Myung and Hong 2015).

Inertial microfluidics where convective forces are present can be used for passive separation processes. Curved micro channels can be designed such that particles of a certain size and mass density are focused at a desired lateral position in a micro channel c.f. (Di Carlo et al. 2007; Özbey et al. 2016). This ease the detection of the desired species, alternatively they can be removed from the fluid for further investigations or purification of the fluid. The channels are geometrically simple, however, with modern lithographic production facilities it is possible to realize channel geometries that go beyond curved micro channels (Gale et al.|2018).

This paper presents a methodology to apply topology optimization to the design of microfluidic devices optimized for individual particle control. This is done by coupling an Eulerian fluid flow solution to a Lagrangian particle tracking method. This enables the designer to optimize a component in which a particle, for instance, follows a specific predefined trajectory which may differ from the streamlines of the flow i.e. the particle is modeled as a finite sized particle with a mass density which may be different from that of the fluid. In this case, it is possible to utilize the inertia effects to make the particle trajectory migrate over the streamlines of the flow. A component design with this ability would over time, produce a stratified flow with locally high / low concentrations of particles. This can be useful in sensor equipment or in a separation process.

The material distribution method for topology optimization was first presented by Bendsøe and Kikuchi (1988) and was introduced in fluid flow optimization by Borrvall and Petersson (2003), optimizing components subjected to steady state Stokes flow. Gersborg-Hansen et al. (2005) extended the methodology to cover laminar 
steady state Navier-Stokes flows. The methodology has later been extended to cover, among other, transient flows (Kreissl et al. 2011, Deng et al. 2011) and turbulent flows (Dilgen et al. 2018a). The modeling of the solid is in the aforementioned references done by using a Brinkman transition flow / lubrication theory based approach, which is also employed in the present work. However, other methods have been presented such as Darcy-Stokes interpolation Guest and Prévost 2006), stabilized Lagrange multiplier approaches(XFEM) (Kre.ssl and Maute 2012) and adaptive no-slip conditions (Behrou et al. 2019).

In terms of microfluidics, multiple different applications have been presented such as designed flexible micro-fluidic devices e.g. manipulative manifolds Kreiss. et al. 2010), microfluidic rectifiers i.e. Tesla micro valves (Deng et al. 2010) and inertia driven dosing units (Andreasen 2017).

Coupled fluid flow and transport problems have been topology optimized in terms of catalytic reactions (Okkels and Bruus 2007) and microfluidic mixing (Andreasen et al. 2009), not to mention the many extensions within convective heat transfer including forced convection e.g. (Yoon 2010, Marck et al. 2013, Dilgen et al. 2018b) and natural convection (Alexandersen et al. 2014, 2016) using the fully coupled Navier-Stokes model.

Topology optimization for mixtures have so-far been focusing on mixing species using Eulerian models of the Navier-Stokes-Brinkman equations with an imposed concentration field. The limitation of the convection-diffusion model used in e.g. Andreasen et al. (2009) is the difficulty in modeling mixtures with very low diffusion. A feasible approach for the non-diffusion case have been shown in Guo et al.2018) where a Lagrangian mapping technique have been applied for the design optimization of a micro mixer.

The problem of optimizing the design of particle control and separation devices have so far not reached a lot of attention. However, Hyun et al. (2017) shows topology optimization of separation devices using the principle of deterministic lateral displacement, in which an array of offset primitives generates a solid grid that induce secondary velocities that eventually make the particles migrate to one side. Since the optimization objective is based on the velocity field, no transported matter is directly modeled.

There are several methods applicable for the modeling of suspended particles. If the concentration is high and many particles of small size are suspended a mixture model might be a good choice. The concentration would then be modeled as a field described by a single variable and the properties of the mixture would result in the simulation of a fluid mixture with spatially

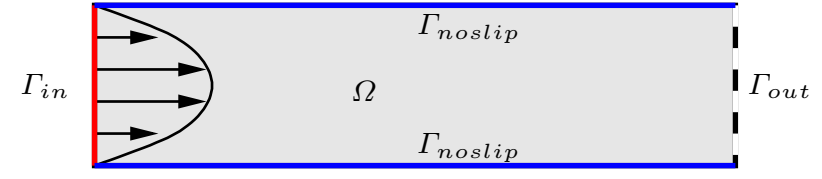

Fig. 1 Sketch for fluid domain. Red boundary denotes inlet, $\Gamma_{i n}$ with a prescribed velocity profile. Dashed black line denotes outlet $\Gamma_{\text {out }}$ with prescribed zero pressure while blue line denotes no-slip wall, $\Gamma_{\text {noslip }}$.

varying properties i.e. effective parameters which would model the flow of the mixture as a whole(Manninen et al. 1996; Lalli et al. 2005). Such a model is fully coupled as the concentration affects the effective properties while the concentration field is advected by the mixture. This is somewhat similar to the case of natural convection problems in convective heat transfer. Such a model may be classified as a macroscopic model as effective parameters are used.

As an alternative, the particles might be modeled individually i.e. using a Lagrangian particle transport model. Here a Lagrangian description of the particle trajectory is used (conservation of momentum) and the interaction with the fluid is included in the equations of motion. The work by Maxey and Riley (1983) presents a set of equations for the accurate modeling of such particle trajectories. As for the mixture model, a Lagrangian model can also be integrated with the fluid flow model, to act as a fully coupled model, introducing the reaction forces from the particle to the fluid flow model.

In this paper, we use a very simplified model assuming that the particles are small and the concentration is very low such that the flow can be modeled undisturbed by the particles. Furthermore, due to the very dilute suspension, the particles are assumed not to interact with each other. The drag from the fluid on the particles is also restricted to the simplest possible drag law, Stokes drag on a sphere. The rotary motion and the possible lift associated to this have also been left out of the study, but are possible future extensions.

The paper is structured such that the flow and particle model is introduced in Section 2 while the numerical model used is introduced in Section 3 . The topology optimization framework and problem definition is presented in Section 4. Numerical results are presented and discussed in Section 5 and finally the paper is concluded in Section 6

\section{Model}

The fluid flow is assumed to be a low speed steady state incompressible flow which can be modeled by the 
Navier-Stokes-Brinkman equations

$-\nabla \cdot \boldsymbol{\sigma}+\rho(\mathbf{u} \cdot \nabla) \mathbf{u}+\alpha(\xi) \mathbf{u}=\mathbf{0}$

in $\Omega$

$\nabla \cdot \mathbf{u}=0$

in $\Omega$

with the stress tensor being defined as

$\boldsymbol{\sigma}=\mu\left(\nabla \mathbf{u}+(\nabla \mathbf{u})^{\mathrm{T}}\right)-\mathbf{I} p$

where $\mathbf{u}$ is the velocity vector, $p$ the pressure, $\rho$ the mass density of the fluid and $\mu$ the absolute viscosity. The term $\alpha(\xi) \mathbf{u}$ is a Brinkman term used to penalize fluid flow locally depending on the spatially varying field $\xi$, which will be determined by the optimization algorithm. Figure 1 shows the generic fluid problem with computational domain $\Omega$ and boundary $\Gamma=\partial \Omega=$ $\Gamma_{\text {in }} \cup \Gamma_{\text {out }} \cup \Gamma_{\text {noslip }}$ on which the following boundary conditions apply:
$\mathbf{u}=\mathbf{0}$
$\mathbf{u}=\mathbf{u}_{0}$
on $\Gamma_{\text {noslip }}$
$p=0$
on $\Gamma_{i n}$
on $\Gamma_{\text {out }}$
$\mathbf{t}=\boldsymbol{\sigma} \cdot \mathbf{n}=\mathbf{0}$
on all $\Gamma$

where $\mathbf{u}_{0}$ is a predefined velocity vector and $\mathbf{t}$ is the traction. The dynamic similarity of flow problems are often characterized by the Reynolds number which for channel flow problems is defined as

$$
R e=\frac{\rho H U}{\mu}
$$

where $H$ is the channel height and the mean flow speed $U$ is given as

$$
U=\frac{\int_{\Gamma_{i n}} \mathbf{u} \cdot \mathbf{n} \mathrm{d} \Gamma}{\left|\Gamma_{i n}\right|}
$$

with $\left|\Gamma_{i n}\right|=\int_{\Gamma_{i n}} 1 \mathrm{~d} \Gamma$ being the length of the inlet.

\subsection{Particle transport}

The motion of each particle, which is being transported by the fluid, can be modeled by the integration of the equations describing linear and angular momentum conservation. However, in order to simplify the modeling, it is assumed that the particles due to their relative small size is not influenced by any rotation around their own axis. Therefore, only the linear momentum conservation is considered here:

$\ddot{\mathbf{x}}_{p} m_{p}-\mathbf{F}_{d}\left(\mathbf{u}\left(\mathbf{x}_{p}\right)-\dot{\mathbf{x}}_{p}\right)=\mathbf{f}$

where $\ddot{\mathbf{x}}_{p}, \dot{\mathbf{x}}_{p}, \mathbf{x}_{p}$ are the particle acceleration, velocity and position, respectively. The spherical particle

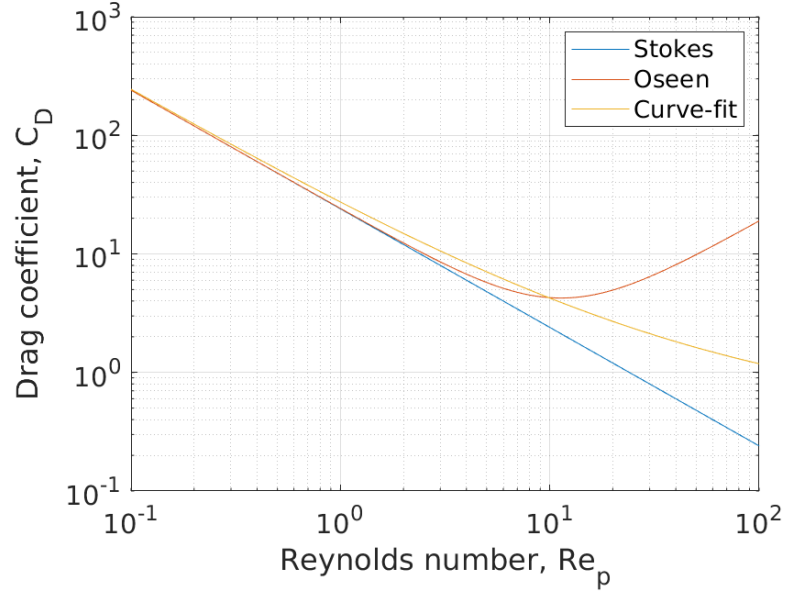

Fig. 2 Drag coefficient vs particle related Reynolds number using Stokes' drag law, the Oseen correction and a curve fit to experimental data(White 1991)

with diameter $d_{p}$ and mass-density $\rho_{p}$ has the mass $m_{p}=\rho_{p} \frac{\pi}{6} d_{p}^{3}$ and $\mathbf{F}_{d}$ is the vector drag force function which is dependent on the particle and fluid velocity, more specifically the difference between the two. The right hand side $\mathbf{f}$ may include other forces acting on the particle for example centrifugal, Coriolis, wall induced lift or inter-particle contact. This is discussed in the next section.

In this work Stokes' drag law is utilized to keep the modeling simple. For a sphere this yield

$\mathbf{F}_{d}(\Delta \mathbf{u})=3 \pi \mu d_{p} \Delta \mathbf{u}$

where $\Delta \mathbf{u}$ is the relative velocity between particle and fluid. Stokes' drag law is valid for very low particle related Reynolds numbers, i.e.

$R e_{p}=\frac{\rho d_{p}\|\Delta \mathbf{u}\|_{2}}{\mu}$

being strictly valid for $R e_{p}<<1$ but predicts well experimentally until $R e_{p} \approx 1$. The drag coefficient can be computed as $C_{D}=2 F / \rho(\Delta u)^{2}$ resulting in $C_{D}=$ $24 / R e_{p}$ for Stokes' flow. For higher $R e_{p}$ the drag is under-predicted and above 20 the flow starts to separate from the rear side as it is visible from Fig. 2 which includes the Stokes' law, Oseen correction and an experimental curve-fit(White 1991).

\subsection{Other forces acting on the particles}

A whole range of other forces could be applied using the presented framework. Some are straightforward while others could be more intriguing. Body forces, such as gravity or fictitious forces from a moving frame of reference i.e. centrifugal and Coriolis, should be relatively 
easy to apply. Wall induced lift forces could potentially be relevant if the size of the particle is non-negligible in comparison to the channel size. That is, the difference in velocity on the near and far side of the sphere might make the particle rotate and generate lift due to the Magnus lift force; this is often referred to as the Saffman effect. In this case, it might be relevant to reconsider modeling the angular momentum balance.

Within microfluidic particle separation an active technique, often utilized, is dielectrophoresis (DEP, see e.g. (Park et al. 2005; Yoon and Park 2010)) where an electric field exerts forces on dielectric particles which change direction based on size and other characteristics allowing for size and material based sorting. This could also be modeled and included in the right hand side as done in Kim et al. (2017) for non-gradient based optimization of electrodes in a DEP based separator. It should, however, be noted that this would also require modeling of the electrical field.

Viscous dissipation in terms of Brownian motion could also be an effect to include in the model. One way of application could be to introduce a random walk at every time-step; alternatively, it could be implemented as a randomized force. In case one would like to ensure robustness of the designed devices, this could be a very relevant addition.

For the particles in this model, we note that they might flow towards the exterior walls and, since they are able to migrate over streamlines, they could potentially hit the wall, which would result in e.g. stiction or reflection. This is not included in this model; however, these effects could also be included and would result in additional contact forces being exerted on the particles.

In addition to the forces acting on the particles mentioned above, the assumptions on rigid and spherical particles might also be discussed. In case the particles are non-rigid e.g. blood cells or other compliant blobs, the fluid-structure-interaction might be influencing the particle movement. The deformability result in an induced lift force which has been reported to depend, among other, on the relation between inertial stress, viscous stress, surface tension and elasticity of the blob c.f. (Chan and Leal 1979; Stan et al. 2013). For modeling non-spherical particles, it might be necessary to consider the equilibrium of angular momentum, including geometry dependent polar moment of inertia and position/angular dependent drag effects due to the nonsphericity.

\subsection{Alternative coupling strategies}

The approach presented assumes that the particles are small. However, this might not be the case for e.g. typical biomedical applications, thus an approach taking the non-negligible size of the particle into account could be considered. One high-fidelity approach is to model the particle and its possible deformation using a fully coupled immersed FSI approach as in Schott et al. (2019) and use a level-set based methodology for optimizing the flow domain e.g. Villanueva and Maute (2017). These approaches are computationally very demanding in comparison to the Lagrangian particle tracking procedure presented here, as the forcing of the particle is modeled directly. A more attractive compromise might be to extend the coupling between the fluid and particle models, such that the reaction force from the particle, is acting on the fluid. In the case where the flow simulation is still assumed steady state, the flow solution must be updated until the distributed reaction forces from the particles converge. Alternatively, the flow solution should also be solved transiently and the flow-particle coupling can be fully integrated in the non-linear solver.

\section{Numerical model}

The numerical solution to the Navier-Stokes-Brinkman equations are obtained by approximating the solution by the use of a stabilized finite element formulation. This allows for use of equal order elements in both velocity and pressure, which is attractive, as it reduce the degrees of freedom per element and in turn allows for higher design resolution.

The equations of (1) and (2) are weakened by multiplication with suitable trail functions (velocity trail function $\mathbf{v}$, pressure trail function $q$, belonging to appropriate function spaces $\mathscr{V}_{0}$ and $\mathscr{P}$, respectively) and integration over the domain $\Omega$ is applied yielding a finite element ready formulation: Find $\mathbf{u} \in \mathscr{V}$ and $p \in \mathscr{P}$ for all $\mathbf{v} \in \mathscr{V}_{0}$ and $q \in \mathscr{P}$ such that

$$
\begin{aligned}
R_{f} & =\int_{\Omega} \nabla \mathbf{v} \cdot\left(\mu\left(\nabla \mathbf{u}+(\nabla \mathbf{u})^{T}\right)-\mathbf{I} p\right) \mathrm{d} \Omega \\
& +\int_{\Omega} \mathbf{v} \cdot \rho(\mathbf{u} \cdot \nabla) \mathbf{u} \mathrm{d} \Omega+\int_{\Omega} \mathbf{v} \cdot \alpha(\xi) \mathbf{u} \mathrm{d} \Omega \\
& -\int_{\Gamma} \mathbf{v} \cdot \mathbf{t} \mathrm{d} \Gamma-\int_{\Omega} q(\nabla \cdot \mathbf{u}) \mathrm{d} \Omega \\
& +\sum_{e=1}^{n_{e l}} \int_{\Omega_{e}} \tau_{S U P G} \mathbf{u} \cdot \nabla \mathbf{v}(\rho \mathbf{u} \cdot \nabla \mathbf{u}+\nabla p+\alpha(\xi) \mathbf{u}) \mathrm{d} \Omega \\
& +\sum_{e=1}^{n_{e l}} \int_{\Omega_{e}} \tau_{P S P G} \nabla q(\mathbf{u} \cdot \nabla \mathbf{u}+\nabla p+\alpha(\xi) \mathbf{u}) \mathrm{d} \Omega=0
\end{aligned}
$$

where the stabilization parameters $\tau_{S U P G}$ and $\tau_{P S P G}$ are obtained based on a simplification of the UGN scheme 
and switching function (Tezduyar et al. 2008):

$$
\begin{aligned}
\tau_{S U P G}=\tau_{P S P G} & =\tau=\left(\frac{1}{\tau_{1}^{2}}+\frac{1}{\tau_{3}^{2}}\right)^{-1 / 2} \\
\tau_{1} & =\frac{2 h_{e}}{\left\|\mathbf{u}_{e}\right\|_{2}}, \quad \tau_{3}=\frac{h_{e}^{2}}{12 \mu}
\end{aligned}
$$

where $h_{e}$ refers to the element side length and $\left\|\mathbf{u}_{e}\right\|_{2}$ is the velocity magnitude evaluated in the center of the element. The equation system is discretized using 4 node quadrilateral elements with bi-linear shape functions. Since equal order elements are used for velocity and pressure PSPG stabilization is needed to circumvent the inf-sub condition. A trade-off from adding PSPG is that the system becomes positive definite, thus more efficient solvers are available. Furthermore, the lower order elements introduce a lower bandwidth of the system matrix allowing a more efficient solution. As the solution might also contain steep solution gradients, an SUPG scheme is added. From the stabilization terms, in which the strong form residual is placed in the parenthesis, it is evident that the diffusion term is left out. This is due to the application of bi-linear elements which are unable to represent 2 nd order derivatives.

The finite element procedure is implemented in a MATLAB framework utilizing the compilation capabilities to ensure a decent solution time despite the need for integrating the element matrices multiple times for every state solution.

The formed residual equation system is solved using a damped Newton scheme with line-search and using a warm-start by utilizing the previous state solution as initial solution vector as in Alexandersen et al. (2014). The Jacobian used in the solution scheme is based on frozen stabilization parameters i.e. it is computed as if the stabilization parameters $(\tau)$ were independent of the velocity.

\subsection{Particle tracking}

For each of the simulated particles the linear momentum equation is integrated in time using an adaptive time step variable order numerical differentiation method available in MATLAB as ode15(Shampine and Reichelt 1997). We prefer an implicit integration scheme as the equation system might be stiff and due to the appealing unconditional stability properties. The integrator uses an adaptive time stepping, however we limit the maximum step size such that intentionally at least one timestep is carried out in every element along the particle trajectory. This is done in order to ensure that any solid element with locally very low velocity will influence the computation of the trajectory. This is crucial since we do not consider the direct contact between solid material and the particles, but rely on the indirect influence on the particle trajectory through the fluid velocity. The maximum time step may be difficult to predict since the design of vary narrow channels introduce dramatically high speeds. A well working maximum time step has been computed as

$\Delta t_{\max }=\frac{1}{2} \frac{h_{e}}{u_{\max }}$

Furthermore, the integration is stopped when the particle leaves the fluid flow domain.

The integration of the trajectory requires the computation of the spatial and velocity dependent drag force. For the computation of the drag force on a particle when passing a specific element, the element shape functions are used to interpolate the velocity based on the current position in the element.

\section{Topology optimization}

The optimization problems considered here are particle control problems with the aim of determining an optimized material distribution such that the particles follow a predefined trajectory or end in a specific part of the domain. In order to control the design, all finite elements are assigned an element-wise constant design value $\xi(\mathbf{x})$ which indicate either solid material $\xi=0$ or fluid $\xi=1$. The integer problem $\xi \in\{0,1\}$ is inherently difficult to solve numerically thus we relax the problem by letting $\xi$ be continuous i.e. $\xi \in[0 ; 1]$.

For flow problems at moderate Reynolds numbers regularization of the design might be needed in order to avoid formation of single element size features. The design is regularized using a smooth Heaviside projection(Wang et al. 2011) yielding a projected design where the filtered design field, $\tilde{\xi}$, and projected design field, $\hat{\xi}$, are obtained as:

$\tilde{\xi}_{i}=\frac{\sum_{j \in N_{e, i}} w\left(\mathbf{x}_{j}^{e}\right) v_{j}^{e} \xi_{j}}{\sum_{j \in N_{e, i}} w\left(\mathbf{x}_{j}^{e}\right) v_{j}^{e}}, \quad w\left(\mathbf{x}_{j}^{e}\right)=R-\left|\mathbf{x}_{j}^{e}-\mathbf{x}_{i}^{e}\right|$

$\hat{\xi}_{i}=\frac{\tanh (\beta \eta)+\tanh \left(\beta\left(\tilde{\xi}_{i}-\eta\right)\right.}{\tanh (\beta \eta)+\tanh (\beta(1-\eta))}$

where $R$ is the filter radius, $N_{e, i}$ the neighborhood and $\mathbf{x}_{i}^{e}$ the spatial coordinates of element $i$. The element area (volume in $3 \mathrm{D}$ ) is $v_{i}$ and the projection parameter $\eta$ describes the projection threshold while $\beta$ describes the steepness and is increased gradually during the optimization procedure.

The projected design is introduced in the governing equations by the inverse permeability $\alpha(\hat{\xi})$ cf. 11$)$ which 
is obtained using an interpolation scheme (Borrvall and Petersson 2003)

$\alpha(\hat{\xi})=\bar{\alpha}+(\underline{\alpha}-\bar{\alpha}) \hat{\xi} \frac{1+q}{\hat{\xi}+q}$

where the lower inverse permeability is vanishing $\underline{\alpha}=0$ while the upper $\bar{\alpha}$ is a relatively large number, typically $\bar{\alpha} \geq 10^{4}$. The penalization parameter, typically $q \leq 1$, determines the weighting of intermediate densities.

When optimizing a device to manipulate the fluid, the cost is very often related to the pressure drop across the component. This is indeed true for the problems treated here. It is expected that by inserting solid material in the flow path, we are able to direct the fluid such that we can manipulate the particle trajectory. Therefore, it is relevant to constrain the pressure drop over the component. We introduce a pressure drop constraint based on the difference between the mean pressures of the inlet and outlet:

$$
\frac{\Delta p}{\gamma \Delta p_{\text {ref }}}-1 \leq 0
$$

and the pressure drop $\Delta p$ is defined as

$\Delta p=\frac{1}{\left|\Gamma_{\text {in }}\right|} \int_{\text {in }} p \mathrm{~d} \Gamma-\frac{1}{\left|\Gamma_{\text {out }}\right|} \int_{\text {out }} p \mathrm{~d} \Gamma$,

and $\Delta p_{r e f}$ is obtained for an empty channel under same conditions and $\gamma$ is the allowed excess pressure multiplier when introducing the design. By limiting the pressure drop, the ability to create very narrow channels is restricted.

The particles are, as mentioned earlier, not directly influenced by the design, and does only feel it through the flow velocities. This means that the optimizer might exploit the finite permeability of the material and take advantage of forcing the particles through areas of intermediate and solid material. In order to try to avoid this, a travel time constraint is introduced that penalizes particles that travel for a longer time than allowed. It penalizes slow particles based on the remaining distance to the outlet boundary. The constraint is formulated as follows:

$$
g=\frac{1}{L} \int_{0}^{T} \mathscr{H}\left(t-\theta t_{\text {empty }}\right)\left(L-x_{1}(t)\right) \mathrm{d} t-\varepsilon \leq 0
$$

where $x_{1}(t)$ is the particle coordinate along the channel and the Heaviside operator is

$\mathscr{H}(s)= \begin{cases}0, & \text { if } s<0 \\ 1, & \text { otherwise }\end{cases}$

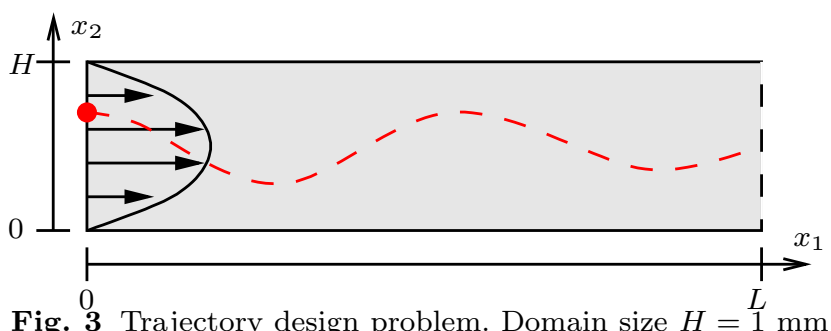

$L=4 \mathrm{~mm}$. Fully developed parabolic inflow with $u_{\max }=5$ $\mathrm{mm} / \mathrm{s}$. Horizontal boundaries are walls with $\mathbf{u}=\mathbf{0}$. Right boundary is outlet $p=0 \mathrm{~Pa}$. Particle seeded at $\mathbf{x}=(0,0.7 H)$ with fluid velocity. Dashed line indicates a target trajectory (sketch).

and $\varepsilon=10^{-4}$ is a small number to ensure that feasibility is possible. The factor $\theta$ relates the travel time of the particle to that of a corresponding full fluid channel $t_{\text {empty }}$. It is noted that this implies that the integration time, $T$, should be larger than the maximum travel time, $T>\theta t_{\text {empty }}$, in order to be useful. For the use with a gradient based optimization algorithm we utilize a smooth implementation of the Heaviside that use a 5 th order polynomial approximation with a transition interval $\Delta s$

$\mathscr{H}^{s}(s, \Delta s)=\left\{\begin{array}{cl}0, & s \leq \Delta s \\ 0.5+0.9375 \frac{s}{\Delta s}-0.625\left(\frac{s}{\Delta s}\right)^{3} & \\ \quad+0.1875\left(\frac{s}{\Delta s}\right)^{5}, & -\Delta s<s<\Delta s \\ 1, & s \geq \Delta s\end{array}\right.$

and here we let $\Delta s=\Delta t_{\max }$ from the time integration scheme.

The specific optimization problems solved are presented in the next sections, however, all problems are solved using the Method of Moving Asymptotes (MMA) (Svanberg 1987) using an external move limit of 0.1 and asymptote initialization, increase and decrease factors being $0.2,1.05,0.65$, respectively.

\subsection{Particle trajectory problem}

The first problem treated is that of making the particle obtain a predefined trajectory i.e. for every point along a channel we may target a certain lateral (vertical) location. Fig. 3 shows a channel with length $L=4 \mathrm{~mm}$ and height $H=1 \mathrm{~mm}$. The upper and lower boundaries are walls with no-slip conditions $\mathbf{u}=\mathbf{0}$ while the left boundary has a fully developed parabolic unidirectional flow profile with maximum velocity, $u_{\max }=5 \mathrm{~mm} / \mathrm{s}$. The right boundary is modeled as an open outlet with prescribed pressure $p=0 \mathrm{~Pa}$. The fluid is water with constant density $\rho=1000 \mathrm{~kg} / \mathrm{m}^{3}$ and absolute viscosity $\mu=10^{-3} \mathrm{~Pa} \cdot \mathrm{s}$. 
As indicated in the figure a particle is initialized at $\mathbf{x}=(0,0.7 H)$ and it is assumed to have the same initial velocity as the surrounding fluid. The solid particle has density $\rho_{p}=1000 \mathrm{~kg} / \mathrm{m}^{3}$ and diameter $d_{p}=10 \mu \mathrm{m}$.

The target trajectory curve is defined relative to $x_{1}$ such that it is independent of time

$x_{2}^{0}=H\left(0.2 \cos \left(\frac{4 \pi x_{1}}{u_{\max }}\right)+0.5\right)$

where $x_{1}$ is the value on the abscissa while $x_{2}^{0}$ is the corresponding target ordinate value. The optimization problem is formulated as a least squares problem (LSQ) in the time domain and subject to a maximum allowed pressure drop and a constraint on the maximum particle travel time

$$
\min _{\boldsymbol{\xi} \in[0 ; 1]} \phi=\ln \left(\frac{1}{H^{2}} \int_{0}^{T}\left(x_{2}(t)-x_{2}^{0}\right)^{2} \mathrm{~d} t\right)
$$

subject to

$$
\begin{aligned}
& R_{f}(\mathbf{s})=0 \\
& R_{p}(\mathbf{x})=0 \\
& \frac{\Delta p}{\gamma \Delta p_{\text {ref }}}-1 \leq 0 \\
& g_{j} \leq 0 \quad \forall j=1, \ldots, N_{p}
\end{aligned}
$$

which is a nested formulation where the residuals of the flow and particle equations are satisfied at every iteration step. For the numerical implementation, it is convenient to scale the objective function by using a logarithm since minimizing the error that has zero lower bound.

This problem is able to demonstrate the ability to optimize the design based on requirements to the particle trajectory. However, it may not demonstrate the full potential of the methodology, which would exploit the inertial characteristics of the particles to make them migrate over streamlines etc.

\subsection{Inertial particle focusing problem}

A more challenging problem is that of focusing particles at a specific lateral location in the flow. Fig. 4 shows the physical domain for the optimization of an inertial focusing device. The fluid enters at the left boundary as a fully developed laminar flow and exits at the right boundary which is open $p=0 \mathrm{~Pa}$. The upper and lower boundaries are modeled with noslip conditions. In this case three particles with mass density similar to steel $\rho_{p}=8000 \mathrm{~kg} / \mathrm{m}^{3}$ and diameter $d_{p}=40 \mu \mathrm{m}$ are seeded from the left boundary at locations $x_{2}=\{0.3 H, 0.5 H, 0.7 H\}$. The objective is make the particles confine in the center of the channel $\left(x_{2}=0.5 H\right)$ at the exit of the device. The design domain is only a part of the physical domain such that it does not influence the inlet and outlet regions. The inlet region has the length corresponding to the channel height while the outlet region is 3 channel heights long. This allows the flow to become fully developed before exiting in the case of using a Reynolds number of 50 . Since the problem is $2 \mathrm{D}$, no streamlines can cross above / below others, thus a fluid particle may always exit in the same lateral position as it entered in case of a fully developed flow. This means that it is only possible to make the device work if the inertia of the particles are utilized to make the trajectories migrate over the streamlines.

The optimization objective is formulated as the sum of error in lateral particle position when the particles exit the computational domain i.e.

$$
\min _{\xi \in[0 ; 1]}
$$$$
\phi=\ln \left(\sum_{p=1}^{N_{p}}\left(\left.x_{2}^{p}\right|_{t=T}-x_{2}^{0}\right)^{2}\right)
$$

subject to

$$
\begin{aligned}
& R_{f}(\mathbf{s})=0 \\
& R_{p}(\mathbf{x})=0 \\
& \frac{\Delta p}{\gamma \Delta p_{\text {ref }}}-1 \leq 0 \\
& g_{j} \leq 0 \quad \forall j=1, \ldots, N_{p}
\end{aligned}
$$

where $N_{p}$ is the number of particles and the lateral outflow position $\left.x_{2}^{p}\right|_{t=T}$ is computed based on the smooth Heaviside function as

$\left.x_{2}^{p}\right|_{t=T} \approx \frac{\int_{0}^{T}\left(H^{s}\left(t-\left(T-\Delta t_{\max }\right), \Delta t_{\max }\right) x_{2}^{p}(t) \mathrm{d} t\right.}{\int_{0}^{T}\left(H^{s}\left(t-\left(T-\Delta t_{\max }\right), \Delta t_{\max }\right) \mathrm{d} t\right.}$

The constraints used are of the same type as for the first problem.

\subsection{Sensitivity analysis}

As outlined for the optimization problems, the objective and constraint functional may depend on the particle motion which is a function of the underlying flow field denoted $\mathbf{s}$ and potentially also directly the physical design $\hat{\xi}$, however, this is not considered here. As the optimization procedure is gradient based, it is depending on the efficient computation of design sensitivities. The general functional can be specified as the following time integral

$\phi=\int_{0}^{T} h(\mathbf{x}(\mathbf{s}(\hat{\xi})), \dot{\mathbf{x}}(\mathbf{s}(\hat{\xi})), \ddot{\mathbf{x}}(\mathbf{s}(\hat{\xi}))) \mathrm{d} t$

In order to compute the sensitivities by the adjoint method (Michaleris et al. 1994, Kang et al. 2006) we 


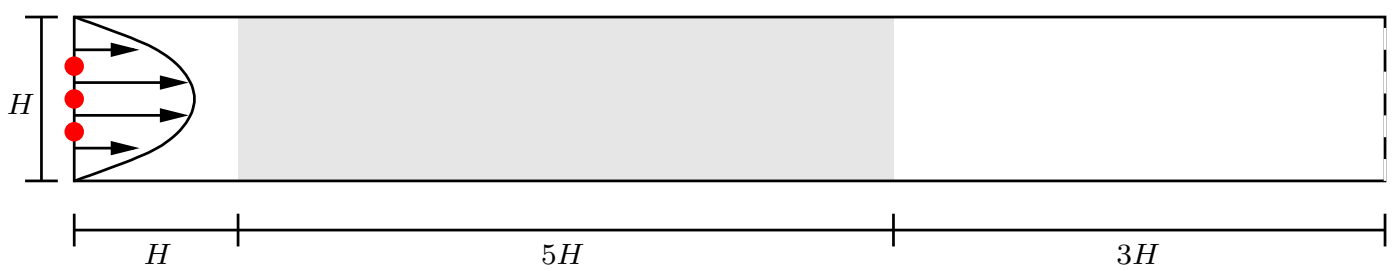

Fig. 4 Problem figure for inertial focusing problem. Grey domain is design domain while inlet and outlet region is fixed fluid. Inlet is parabolic profile, outlet is prescribed pressure $p=0$ and horizontal boundaries are no-slip. Three particles are seeded at the inlet (red dots) in vertical position $x_{2}=\{0.3 H, 0.5 H, 0.7 H\}$ where $H=1 \mathrm{~mm}$. Particle target is vertical position $x_{2}^{0}=0.5 H$ on the outlet.

define the Lagrangian function by augmenting the objective with a product of an adjoint vector and the residual of the state equations, flow and particle, respectively. The particle problem is transient, and we consider the steady state flow problem, which simplifies the adjoint formulation significantly for this part of the problem. The Lagrangian is constructed by adding two zero terms to the objective which yields:

$\mathcal{L}=\int_{0}^{T} h(\mathbf{x}, \dot{\mathbf{x}}, \ddot{\mathbf{x}}) \mathrm{d} t+\boldsymbol{\lambda}_{f}^{\mathrm{T}} R_{f}+\int_{0}^{T} \boldsymbol{\lambda}_{p}^{\mathrm{T}} R_{p} \mathrm{~d} t$

where $\boldsymbol{\lambda}_{f}$ and $\boldsymbol{\lambda}_{p}$ are the adjoint vectors associated to the fluid and particle problems, respectively. They have the same dimensions as the corresponding state vectors. In the following the chain rule is utilized and it is emphasized that the objective functional is assumed to depend explicitly on the particle variables, $\mathbf{x}, \dot{\mathbf{x}}, \ddot{\mathbf{x}}$ and only implicitly on the flow s and design $\hat{\xi}$. Differentiating the Lagrangian yield

$$
\begin{aligned}
\frac{\mathrm{d} \mathcal{L}}{\mathrm{d} \hat{\xi}} & =\int_{0}^{T}\left(\frac{\partial h}{\partial \mathbf{x}} \frac{\mathrm{d} \mathbf{x}}{\mathrm{d} \hat{\xi}}+\frac{\partial h}{\partial \dot{\mathbf{x}}} \frac{\mathrm{d} \dot{\mathbf{x}}}{\mathrm{d} \hat{\xi}}+\frac{\partial h}{\partial \ddot{\mathbf{x}}} \frac{\mathrm{d} \ddot{\mathbf{x}}}{\mathrm{d} \hat{\xi}}\right) \mathrm{d} t \\
& +\boldsymbol{\lambda}_{f}^{T}\left(\frac{\partial R_{f}}{\partial \hat{\xi}}+\frac{\partial R_{f}}{\partial \mathbf{s}} \frac{\mathrm{d} \mathbf{s}}{\mathrm{d} \hat{\xi}}\right) \\
& +\int_{0}^{T} \lambda_{p}^{T}\left(\frac{\partial R_{p}}{\partial \mathbf{s}} \frac{\mathrm{d} \mathbf{s}}{\mathrm{d} \hat{\xi}}+\frac{\partial R_{p}}{\partial \mathbf{x}} \frac{\mathrm{d} \mathbf{x}}{\mathrm{d} \hat{\xi}}+\frac{\partial R_{p}}{\partial \dot{\mathbf{x}}} \frac{\mathrm{d} \dot{\mathbf{x}}}{\mathrm{d} \hat{\xi}}+\frac{\partial R_{p}}{\partial \ddot{\mathbf{x}}} \frac{\mathrm{d} \ddot{\mathbf{x}}}{\mathrm{d} \hat{\xi}}\right)
\end{aligned}
$$

We may refer to the above function based on splitting it depending on if a term is multiplied by the derivative of particle position, velocity and acceleration, here denoted $\mathbf{X}$ as a common, w.r.t. design, and the derivative of the flow state w.r.t. design or simply is the direct derivative w.r.t. the design:

$$
\frac{\mathrm{d} \mathcal{L}}{\mathrm{d} \hat{\xi}}=\frac{\partial \mathcal{L}}{\partial \mathbf{X}} \frac{\mathrm{d} \mathbf{X}}{\mathrm{d} \hat{\xi}}+\frac{\partial \mathcal{L}}{\partial \mathbf{s}} \frac{\mathrm{d} \mathbf{s}}{\mathrm{d} \hat{\xi}}+\frac{\partial \mathcal{L}}{\partial \hat{\xi}}
$$

\subsection{Sensitivity analysis for particles}

Collecting the terms from the above equation that involve the particle state derivatives i.e. first term of 19 yield

$\int_{0}^{T}\left(\frac{\partial h}{\partial \mathbf{x}} \frac{\mathrm{d} \mathbf{x}}{\mathrm{d} \hat{\xi}}+\frac{\partial h}{\partial \dot{\mathbf{x}}} \frac{\mathrm{d} \dot{\mathbf{x}}}{\mathrm{d} \hat{\xi}}+\frac{\partial h}{\partial \ddot{\mathbf{x}}} \frac{\mathrm{d} \ddot{\mathbf{x}}}{\mathrm{d} \hat{\xi}}\right) \mathrm{d} t$
$+\int_{0}^{T} \boldsymbol{\lambda}_{p}^{T}\left(\frac{\partial R_{p}}{\partial \mathbf{x}} \frac{\mathrm{d} \mathbf{x}}{\mathrm{d} \hat{\xi}}+\frac{\partial R_{p}}{\partial \dot{\mathbf{x}}} \frac{\mathrm{d} \dot{\mathbf{x}}}{\mathrm{d} \hat{\xi}}+\frac{\partial R_{p}}{\partial \ddot{\mathbf{x}}} \frac{\mathrm{d} \ddot{\mathbf{x}}}{\mathrm{d} \hat{\xi}}\right) \mathrm{d} t$

Following the procedure as outlined in e.g. Kang et al. (2006) and applying partial integration to the residual terms being differentiated wrt. acceleration yield

$$
\begin{aligned}
& \int_{0}^{T} \boldsymbol{\lambda}_{p}^{T} \frac{\partial R_{p}}{\partial \ddot{\mathbf{x}}} \frac{\mathrm{d} \ddot{\mathbf{x}}}{\mathrm{d} \hat{\xi}} \mathrm{d} t=\left[\boldsymbol{\lambda}_{p}^{T} \frac{\partial R_{p}}{\partial \ddot{\mathbf{x}}} \frac{\mathrm{d} \dot{\mathbf{x}}}{\mathrm{d} \hat{\xi}}\right]_{0}^{T}-\int_{0}^{T} \dot{\boldsymbol{\lambda}}_{p}^{T} \frac{\partial R_{p}}{\partial \ddot{\mathbf{x}}} \frac{\mathrm{d} \dot{\mathbf{x}}}{\mathrm{d} \hat{\xi}} \\
& =\left[\boldsymbol{\lambda}_{p}^{T} \frac{\partial R_{p}}{\partial \ddot{\mathbf{x}}} \frac{\mathrm{d} \dot{\mathbf{x}}}{\mathrm{d} \hat{\xi}}\right]_{0}^{T}-\left[\dot{\lambda}_{p}^{T} \frac{\partial R_{p}}{\partial \ddot{\mathbf{x}}} \frac{\mathrm{d} \mathbf{x}}{\mathrm{d} \hat{\xi}}\right]_{0}^{T}+\int_{0}^{T} \ddot{\boldsymbol{\lambda}}_{p}^{T} \frac{\partial R_{p}}{\partial \ddot{\mathbf{x}}} \frac{\mathrm{d} \mathbf{x}}{\mathrm{d} \hat{\xi}}
\end{aligned}
$$

while those including the derivative wrt. velocity yield

$$
\int_{0}^{T} \boldsymbol{\lambda}_{p}^{T} \frac{\partial R_{p}}{\partial \dot{\mathbf{x}}} \frac{\mathrm{d} \dot{\mathbf{x}}}{\mathrm{d} \hat{\xi}} \mathrm{d} t=\left[\boldsymbol{\lambda}_{p}^{T} \frac{\partial R_{p}}{\partial \dot{\mathbf{x}}} \frac{\mathrm{d} \mathbf{x}}{\mathrm{d} \hat{\xi}}\right]_{0}^{T}-\int_{0}^{T} \dot{\boldsymbol{\lambda}}_{p}^{T} \frac{\partial R_{p}}{\partial \dot{\mathbf{x}}} \frac{\mathrm{d} \mathbf{x}}{\mathrm{d} \hat{\xi}}
$$

For the functional (e.g. objective function) terms the application of integration by parts gives

$\mathrm{d} t \quad \int_{0}^{T} \frac{\partial h}{\partial \ddot{\mathbf{x}}} \frac{\mathrm{d} \ddot{\mathbf{x}}}{\mathrm{d} \hat{\xi}} \mathrm{d} t=\left[\frac{\partial h}{\partial \ddot{\mathbf{x}}} \frac{\mathrm{d} \ddot{\mathbf{x}}}{\mathrm{d} \hat{\xi}}\right]_{0}^{T}-\int_{0}^{T} \frac{\mathrm{d}}{\mathrm{d} t} \frac{\partial h}{\partial \ddot{\mathbf{x}}} \frac{\mathrm{d} \dot{\mathbf{x}}}{\mathrm{d} \hat{\xi}} \mathrm{d} t$
$=\left[\frac{\partial h}{\partial \ddot{x}} \frac{\mathrm{d} \ddot{\mathbf{x}}}{\mathrm{d} \hat{\xi}}\right]_{0}^{T}-\left[\frac{\mathrm{d}}{\mathrm{d} t} \frac{\partial h}{\partial \ddot{\mathbf{x}}} \frac{\mathrm{d} \mathbf{x}}{\mathrm{d} \hat{\xi}}\right]_{0}^{T}-\int_{0}^{T} \frac{\mathrm{d}^{2}}{\mathrm{~d} t^{2}} \frac{\partial h}{\partial \ddot{\mathbf{x}}} \frac{\mathrm{d} \mathbf{x}}{\mathrm{d} \hat{\xi}} \mathrm{d} t$

and finally

$\int_{0}^{T} \frac{\partial h}{\partial \dot{\mathbf{x}}} \frac{\mathrm{d} \dot{\mathbf{x}}}{\mathrm{d} \hat{\xi}}=\left[\frac{\partial h}{\partial \dot{\mathbf{x}}} \frac{\mathrm{d} \mathbf{x}}{\mathrm{d} \hat{\xi}}\right]_{0}^{T}-\left[\frac{\mathrm{d}}{\mathrm{d} t} \frac{\partial h}{\partial \dot{\mathbf{x}}} \frac{\mathrm{d} \mathbf{x}}{\mathrm{d} \hat{\xi}}\right]_{0}^{T}$

The adjoint variables $\boldsymbol{\lambda}_{p}$ can now be obtained from the transient adjoint problem:

$$
\begin{aligned}
\left(\frac{\partial R_{p}}{\partial \ddot{\mathbf{x}}}\right)^{\mathrm{T}} \ddot{\boldsymbol{\lambda}}_{p} & -\left(\frac{\partial R_{p}}{\partial \dot{\mathbf{x}}}\right)^{\mathrm{T}} \dot{\boldsymbol{\lambda}}_{p}+\left(\frac{\partial R_{p}}{\partial \mathbf{x}}\right)^{\mathrm{T}} \boldsymbol{\lambda}_{p} \\
& =\left(-\frac{\mathrm{d}^{2}}{\mathrm{~d} t^{2}} \frac{\partial h}{\partial \ddot{\mathbf{x}}}+\frac{\mathrm{d}}{\mathrm{d} t} \frac{\partial h}{\partial \dot{\mathbf{x}}}-\frac{\partial h}{\partial \mathbf{x}}\right)^{\mathrm{T}}
\end{aligned}
$$


The initial conditions for the particles are given a priori and are independent from the design i.e.

$\left.\frac{\mathrm{d} \mathbf{x}}{\mathrm{d} \hat{\xi}}\right|_{t=0}=0 \quad$ and $\left.\quad \frac{\mathrm{d} \dot{\mathbf{x}}}{\mathrm{d} \hat{\xi}}\right|_{t=0}=0$

Furthermore, none of the objective and constraint functions presented depend on particle velocity, $\dot{\mathbf{x}}$, and acceleration, $\ddot{\mathbf{x}}$, thus the time evaluated terms of $230,224,225$ and (26) reduce to the terminal conditions for the adjoint problem

$\lambda_{p}(T)=0, \quad \dot{\lambda}_{p}(T)=0$

We solve this terminal value problem by shifting time $\tau=T-t$ such that an initial value problem appear. This makes it possible to use the same integration scheme as for the forward problem, however, one needs to pay attention to the functional specific and time-dependent loads that apply and possibly vary with time and/or position.

As the design sensitivities can be decomposed as $\frac{\mathrm{d} \phi}{\mathrm{d} \hat{\xi}}=\frac{\mathrm{d} \phi}{\mathrm{d} \mathbf{s}} \frac{\mathrm{d} \mathbf{s}}{\mathrm{d} \hat{\xi}}$ the sensitivities w.r.t. the flow state can the be obtained as

$\frac{\mathrm{d} \phi}{\mathrm{d} \mathbf{s}}=\int_{0}^{T}\left(\lambda_{p}^{T} \frac{\partial R_{p}}{\partial \mathbf{s}}\right) \mathrm{d} t$

alternatively the sensitivity analysis can proceed to find the total design sensitivities.

\subsection{Sensitivity analysis for flow}

Based on the second term in 19 i.e the terms that is multiplied by the derivatives of the flow state w.r.t. we may obtain another adjoint problem by letting the first factor be zero to avoid evaluating $\frac{\mathrm{ds}}{\mathrm{d} \hat{\xi}}$ i.e.:

$$
\begin{gathered}
\left(\boldsymbol{\lambda}_{f}^{\mathrm{T}} \frac{\partial R_{f}}{\partial \mathbf{s}}+\int_{0}^{T} \boldsymbol{\lambda}_{p}^{\mathrm{T}}\left(\frac{\partial R_{p}}{\partial \mathbf{s}}\right) \mathrm{d} t\right) \frac{\mathrm{d} \mathbf{s}}{\mathrm{d} \hat{\xi}} \Rightarrow \\
\left(\frac{\partial R_{f}}{\partial \mathbf{s}}\right)^{\mathrm{T}} \boldsymbol{\lambda}_{f}=-\int_{0}^{T}\left(\frac{\partial R_{p}}{\partial \mathbf{s}}\right)^{\mathrm{T}} \boldsymbol{\lambda}_{p} \mathrm{~d} t
\end{gathered}
$$

where the right hand side was obtained in the sensitivity analysis for the particle problem just above. The sensitivities may afterwards be evaluated as

$\frac{\mathrm{d} \phi}{\mathrm{d} \hat{\xi}}=\frac{\mathrm{d} \mathcal{L}}{\mathrm{d} \hat{\xi}}=\lambda_{f}^{\mathrm{T}} \frac{\partial R_{f}}{\partial \hat{\xi}}$

based on the third term in (19). We may remark that this sequence of adjoint methods where the sensitivities from the particle problem enter in the adjoint flow problem is only possible as long as the particles are not influencing the flow. In that case the problem must be
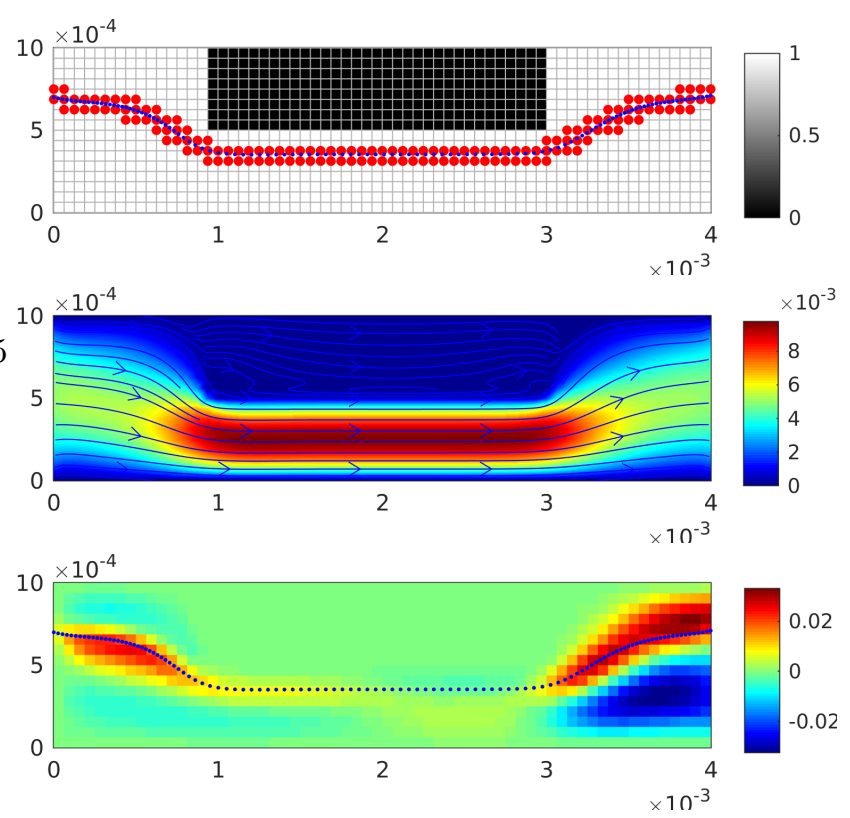

Fig. 5 The figures show the very localized sensitivity contributions from the particle (upper figure). The middle figure shows the velocity magnitude for flow under a box and the lower figure shows the total sensitivity distribution for the target trajectory objective.

considered fully coupled as e.g. when considering natural convection problems (Alexandersen et al. 2014) and solved simultaneously.

The loading of the adjoint flow problem is based on the particle trajectory and since the velocities used in the Lagrangian method is obtained using the local support of the finite element shape functions, the particle trajectory only depends on nodes related to elements that lie on the particle path. This introduce a loading along the trajectory of the particle. An example of the very localized sensitivities obtained when tracking a particle is visualized in Fig. 5 . The design problem is that of following a target trajectory as shown in Fig. 3. In the domain, a solid area is indicated by black color and the coarse 64 by 16 mesh is shown for clarity. The red nodes are non-zero particle problem sensitivities i.e. these are used for the load of the adjoint flow problem. The center figure shows the velocity magnitude field and the lower figure shows the total sensitivity i.e. $\frac{d \phi}{d \hat{\xi}}$. Since the objective is in this case depending on the entire particle trajectory, we see that the sensitivity is high around the trajectory. For problems depending on the lateral particle outlet position $\left.x_{2}\right|_{t=T}$ the sensitivities seem to be more intense in the outlet region. 


\subsection{Chain-rule for projection filter}

The sensitivities were all obtained for the physical field $\hat{\xi}$, however, since the optimizer uses the mathematical field $\xi$, sensitivities need to be updated accordingly:

$\frac{\partial \phi}{\partial \xi_{i}}=\sum_{j \in N_{e, i}} \frac{\partial \phi}{\partial \hat{\xi}_{j}} \frac{\partial \hat{\xi}_{j}}{\partial \tilde{\xi}_{j}} \frac{\partial \tilde{\xi}_{j}}{\partial \xi_{i}}$

\subsection{Robust formulation}

The introduction of the Heaviside projection in the design parameterization allows for easy integration of the so-called robust formulation(Wang et al. 2011). For some problems, the intermediate valued design variables seem to govern the design process due to low intrinsic penalization in the problem. The optimizer may possibly take advantage of the very detailed control, which can be applied when relying on intermediate values. As the main goal is to achieve 0-1 designs that represent clear geometries, one remedy is to introduce the robust formulation in which multiple design realizations are evaluated and optimized. For the problem of sec. 4.1 this may be stated as a min-max problem:

$$
\begin{aligned}
\min _{\boldsymbol{\xi} \in[0 ; 1]} \max _{i \in\{e, b, d\}} & \phi^{i}=\ln \left(\frac{1}{H^{2}} \int_{0}^{T}\left(x_{2}^{i}(t)-x_{2}^{0}\right)^{2} \mathrm{~d} t\right) \\
\text { subject to } & R_{f}(\mathbf{s})^{i}=0 \text { for } i \in\{e, b, d\} \\
& R_{p}(\mathbf{x})^{i}=0 \text { for } i \in\{e, b, d\} \\
& \frac{\Delta p^{i}}{\gamma \Delta p_{\text {ref }}}-1 \leq 0 \text { for } i \in\{e, b, d\} \\
& g_{j} \leq 0 \quad \forall j=1, \ldots, N_{p}
\end{aligned}
$$

where $e, b, d$ corresponds to an eroded, blue-print and dilated design, respectively. The design realizations are achieved by changing the offset parameter $\eta$ in the Heaviside projection. The min-max formulation might be solved using the bound formulation(Olhoff 1989) which is supported by MMA.

Finally, it should be noted that an alternative strategy could be to explicitly penalize the intermediate design variables by augmenting e.g. $\sum_{e=1}^{n_{e l}}\left(1-\hat{\xi}_{e}\right) \hat{\xi}_{e}$ to the objective function using an appropriate weight. This approach is in general quite sensitive to the weighting, thus applying it as a sort of post-processing step may be more useful.

\section{Results}

The result section at first presents a verification of the particle integration method by comparing to a commercial software and a small study of particle trajectories

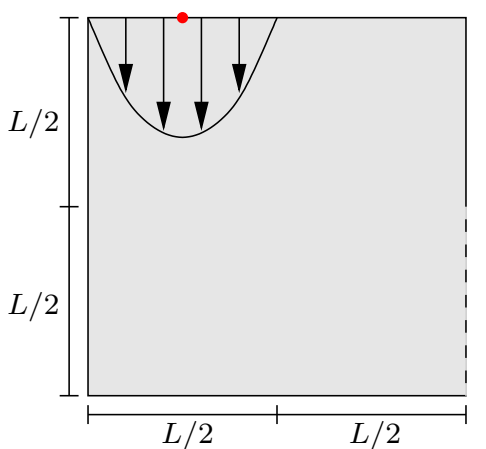

Fig. 6 Problem setup for the verification and study of particle streamline migration. Square cavity with side length $L=4 \mathrm{~mm}$. Parabolic inflow $u_{\max }=50 \mathrm{~mm} / \mathrm{s}$. Outlet boundary $p_{\text {out }}=0 \mathrm{~Pa}$. No-slip boundary conditions on remaining boundary.

migrating over streamlines. Next the results of the two optimization problems are presented and the most influential parameters are investigated and features discussed.

\subsection{Verification of particle tracking integration}

Using the multiphysics simulation software COMSOL 5.4 we verify that the particle integration is accurate enough. The flow through a square cavity with inlet at the left half of the top edge and outlet at the lower part of the right edge is investigated c.f. Fig. 6. The inlet is fully developed and the outlet is modeled as an open boundary with uniform pressure $p=0 \mathrm{~Pa}$. A particle is released in the center part of the inlet with same initial velocity as the fluid, i.e. it is entering with the fluid. For a particle with mass density $\rho_{p}=$ $8000 \mathrm{~kg} / \mathrm{m}^{3}$ and diameter $d_{p}=40 \mu \mathrm{m}$ Fig. 7 shows the particle trajectories and relative error by comparison to the trajectory obtained using COMSOL 5.4. It is seen from the coordinate vs. time plot that the particles travel on the same path. By inspecting the plot of the relative error, it is seen that it is below $5 \times 10^{-5}$ which is indeed small and related to the integration accuracy of the time integrator. It should also be noted that the COMSOL result is obtained using a generalized alpha time integrator.

In addition to the above particle an extra particle of density $\rho_{p}=19200 \mathrm{~kg} / \mathrm{m}^{3}$ (Gold) is simulated and Fig. 8 shows the obtained trajectories along with a streamline integrated from the initial particle location. It is apparent that the particles are migrating over streamlines due to the higher density than the surrounding fluid, which is magnified in the zoom to the right. The particle with $\rho_{p}=8000 \mathrm{~kg} / \mathrm{m}^{3}$ exits $0.03 \mathrm{~mm}$ further down than the corresponding streamline while the particle with $\rho_{p}=19200 \mathrm{~kg} / \mathrm{m}^{3}$ exits $0.08 \mathrm{~mm}$ lower. 

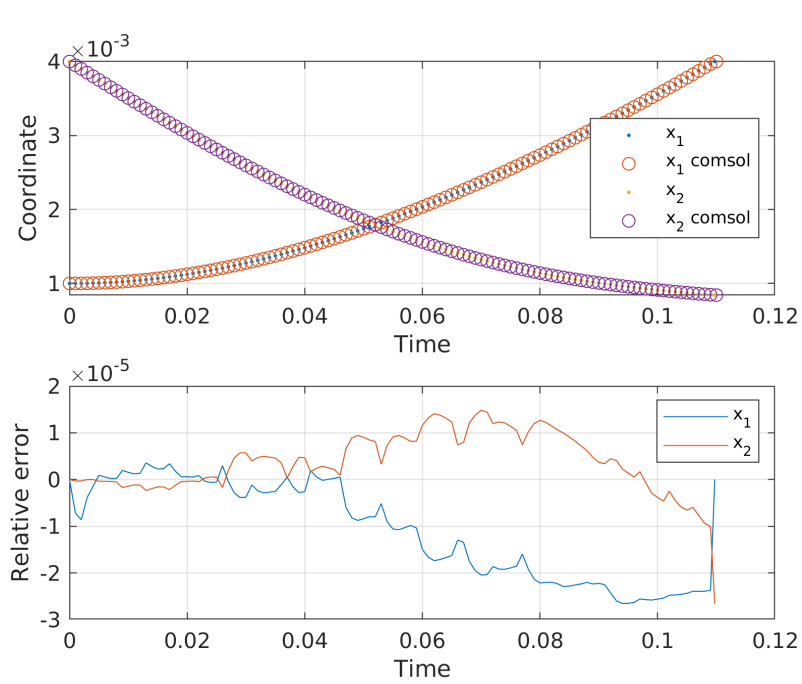

Fig. 7 Upper: Particle trajectory vs. time for corner flow problem integrated with the present framework and COMSOL. Lower: Relative error in comparison to COMSOL. Fluid density $\rho=1000 \mathrm{~kg} / \mathrm{m}^{3}$ and viscosity $\mu=0.001 P a . s$. Particle density $\rho_{p}=8000 \mathrm{~kg} / \mathrm{m}^{3}$ and diameter $d_{p}=40 \mu \mathrm{m}$
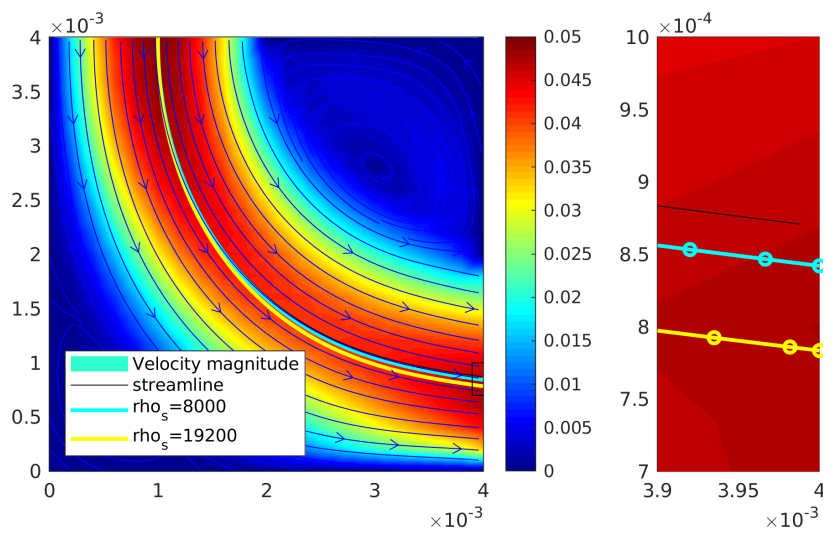

Fig. 8 Left: Particle trajectories for corner flow problem width fluid density $\rho=1000 \mathrm{~kg} / \mathrm{m}^{3}$ and viscosity $\mu=0.001$ Pa.s. Particle diameter $d_{p}=40, \mu \mathrm{m}$ simulated on a $20 \times 20$ elements mesh. Right: Close-up of particle trajectories near the outlet. Black streamline, cyan trajectory $\rho_{p}=8000 \mathrm{~kg} / \mathrm{m}^{3}$, yellow trajectory $\rho_{p}=19200 \mathrm{~kg} / \mathrm{m}^{3}$

\subsection{Optimization for target trajectory}

For the optimization of a flow component designed for a specific particle trajectory, c.f. Sec. 4.1, a result is seen in Fig. 9. The design is shown in grey-scale with desired particle trajectory as a red dashed line and the actual trajectory shown as a series of blue dots indicating the integration points along the trajectory. It is clearly seen that a binary design is obtained in most of the design domain. There is, however, a small region attaining grey intermediate design resulting in a cusp on the trajectory. This may be contributed the vanishing penalization when enforcing a pressure drop constraint
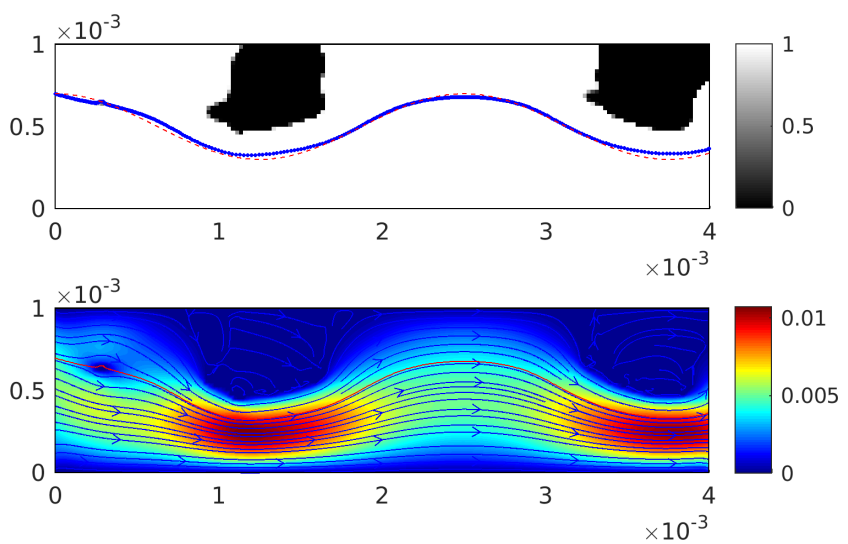

Fig. 9 Upper: Optimized design for target trajectory flow optimization. Red dashed curve is target. Dotted blue points is particle trajectory. Lower: Velocity magnitude plot with streamlines. Red curve is particle trajectory. $\gamma=5,160 \times 40$ elements.

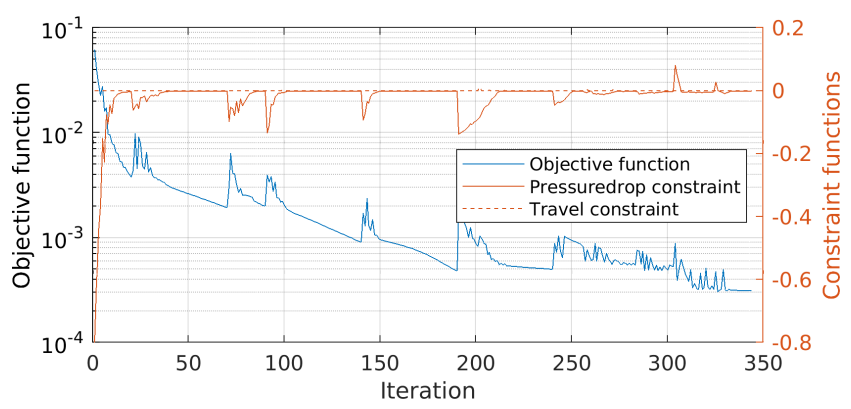

Fig. 10 Optimization history for the design in Fig. 9 Blue line shows objective function. Red solid line is pressure drop constraint and dashed-red line is travel constraint. Minor peaks appear due to continuation of the $\beta$-parameter. Final objective: $\phi=-8.06$. Pressure drop constraint is active. Travel time constraint not active $(\theta=1.5)$

instead of a volume constraint and the fact that a transition zone remains even when the projection is applied. The optimizer is not fully able to adapt the particle trajectory to the target trajectory which is due to the limitation on the pressure drop which is here $\gamma=5$. From the lower plot, it is evident that the inertia of the particle is different from the surrounding fluid such that it is able to migrate over several streamlines along the flow component. Fig. 10 shows the evolution in objective and constraint functions during the optimization procedure. The problem behaves very nicely and small oscillations are visible due to the $\beta$-continuation.

The ability to attain the desired trajectory is closely related to the allowed pressure drop, which is clear when inspecting the obtained designs for various $\gamma$ in Fig. 11 . It is seen that when the pressure drop is limiting the design we obtain binary designs while the designs obtained for e.g. $\gamma=10$ is seen to contain many intermediate densities despite the projection. This can be contributed the inactive pressure constraint as it tends 


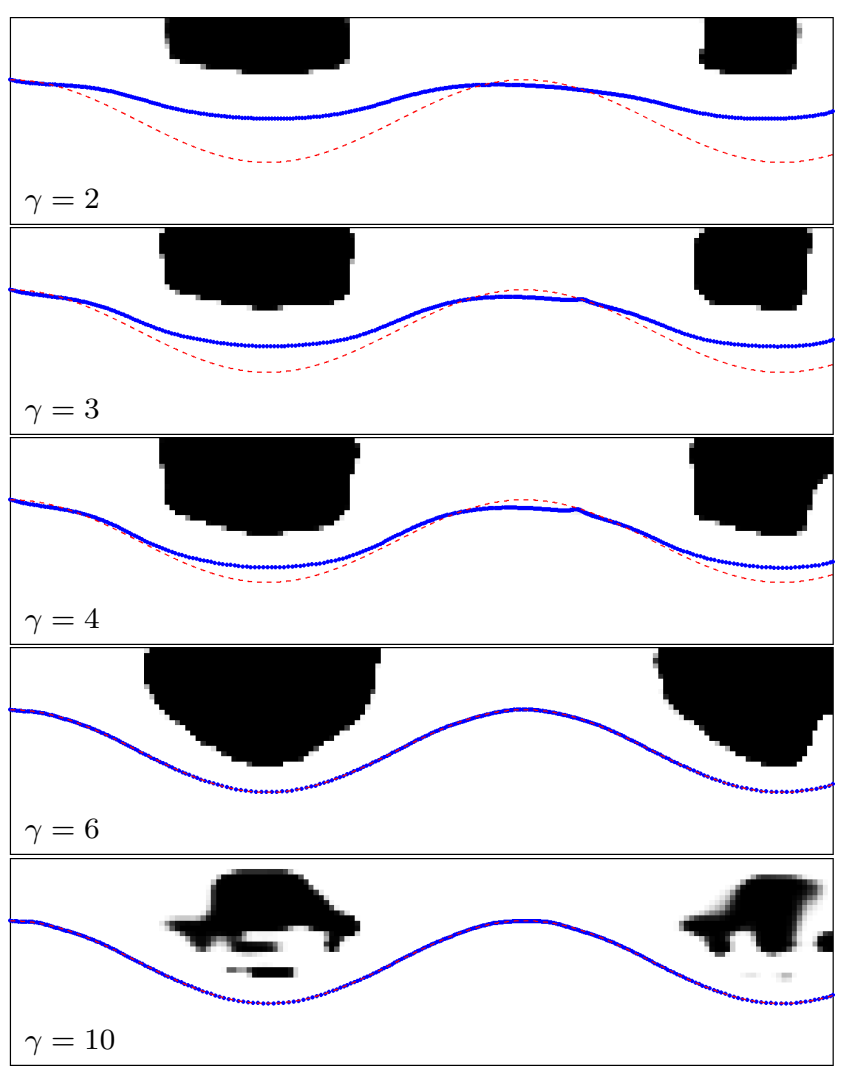

Fig. 11 Designs obtained for varying the allowed pressure drop. Starting from top $\gamma=\{2,3,4,6,10\}$ for $\rho_{p}=1000 \mathrm{~kg} / \mathrm{m}^{3}$. Travel time constraint set to initial particle travel time i.e. $\theta=1$. Objective values: $\phi=$ $\{-4.35,-5.67,-6.90,-13.50,-13.29\}$

to clean up the design. The pressure drop constraint may reduce the scattering in the design and ensure that there is a flow path; however, it may not ensure that the particle does not encounter solid obstacles on its way through the component. The adjustment of the travel constraint may control this feature. This is visible from Fig. 12 where the travel time constraint have been omitted. The optimizer tends to control the trajectory of the particle by putting material very close to or directly where the particle is flowing. In combination with the projection this eventually, lead to solid material being placed directly in the desired trajectory. One may at times obtain useful designs without the constraint; however, it helps regularizing the design during the optimization process and limits the amount of solid islands that the particle must pass.

By tightening the travel constraint, we see that the optimizer needs to synthesize a design where the flow speed is generally higher. This in turn cause trouble depending on the allowed pressure drop as shown in Fig. 13 where the travel constraint is varied. For the first two cases, the travel time constraint ensures at first a narrow channel to meet the requirement, however

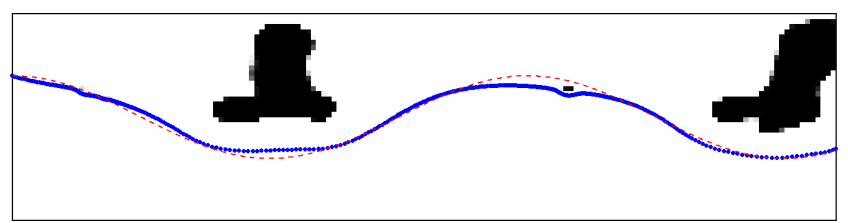

Fig. 12 Design obtained without travel time constraint for allowed pressure drop $\gamma=6$ and $\rho_{p}=1000 \mathrm{~kg} / \mathrm{m}^{3}$. Islands of intermediate and solid material placed directly on the target trajectory. $\phi=-6.99$

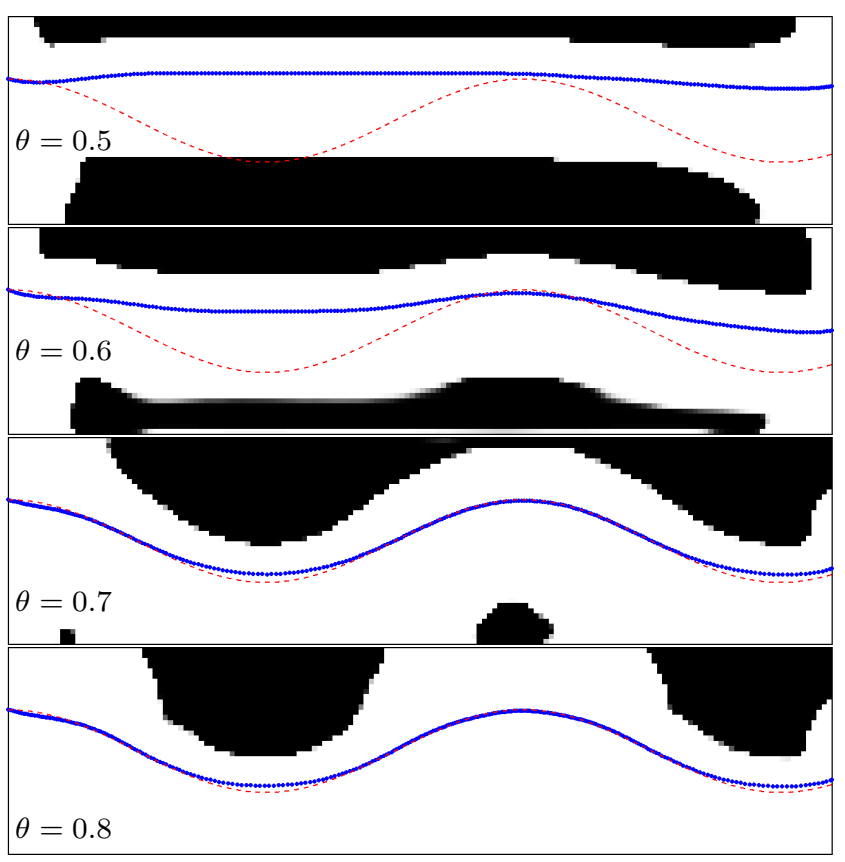

Fig. 13 A study of the travel time constraint influence. From above the allowed time fraction is varied as $\theta=$ $\{0.5,0.6,0.7,0.8\}$. Allowed pressure drop: $\gamma=5$. Objective values: $\phi=\{-3.23,-4.25,-8.50,-9.09\}$

since the pressure drop constraint is also active, there are clear restrictions to the channel curvature. During the entire optimization process, the two upper design cases have active travel and pressure drop constraints.

For larger travel times the constraint have forced the optimizer to prioritize material placement in the early design process. As the continuation scheme for $\beta$ increase the steepness of projection, the pressure drop constraint becomes inactive and gives the optimizer more freedom, which is visible by small material inclusions containing grey scale.

Some of the design cases for varying $\gamma$ have been optimized using a refined mesh i.e. $320 \times 80$ elements and $R=6$ elements and showed similar results, but for consistency all problems have been shown using the same resolution. 


\subsubsection{Notes about the optimization procedure}

The optimization procedure includes a continuation of the projection parameter $\beta$ and the maximum inverse permeability $\bar{\alpha}$. The value of $\beta$ is increased during the optimization according to the sequence

$\beta=\{0.1,1.1,2.1,3.1,4,8,16,32,64\}$ if the constraints are satisfied and 50 iterations have passed since last change. Alternatively, it is increased if the MMA design change between iteration $\mathrm{k}$ and $\mathrm{k}+1,\left\|\xi_{k+1}-\xi_{k+1}\right\|_{\infty}$, is below $1 \%$. All optimizations start using $\bar{\alpha}=10^{4}$ and when $\beta=64$ is reached, the continuation is shifted to $\bar{\alpha}$ which is multiplied by a factor 10 it until $\bar{\alpha}=10^{6}$. Hereafter, the optimization continue until either the design change drop below $1 \%$ or 500 iterations have been reached. The penalization parameter is maintained constant $q=1$ for all designs.

It has been observed that the design changes with the mentioned settings for MMA are relatively small and thus the design changes rather slowly. This can, however, be sped up by adjusting the aggressiveness MMA i.e. changing asymptote initialization, increase and decrease factors to $0.5,1.2$ and 0.7 , respectively, instead of the relatively conservative settings applied here. There are, however, clear drawbacks by doing so. While optimizing swiftly during the initial phase of the optimization, the latter phase may become very oscillatory due to the very localized and high sensitives induced on the trajectory by changing an upstream element dramatically. By introducing a relatively large change, the sensitivity information is not adequate as the step induce a very large change in trajectory, which is far beyond the validity of the sensitivity information. This has been investigated by carefully inspecting the convergence of finite difference sensitivities for some elements along the flow path that show a good quality of sensitivities for small perturbations. This observation is true for the physical problem itself, even without adding the Heaviside projection. Adding this and using a large $\beta$-value only amplifies the problem. A feasible approach might be to allow large design changes in the beginning and reducing them either by move limit or by restrictions on optimizer as a part of the continuation scheme.

\subsubsection{Eliminating intermediate design variables by the robust formulation}

In section 4.7 the robust formulation was introduced and as noted above some of the designs contain small material inclusions or islands that may seem to be an artifact of the formulation rather than an objective gain. The results in e.g. Fig. 11 obtained for $\gamma=\{3,4\}$ show a very weak kink in the trajectories which may

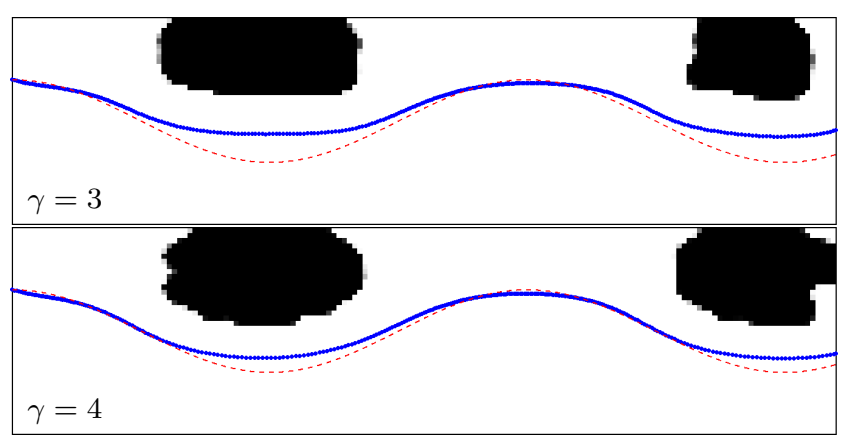

Fig. 14 Designs for $\gamma=\{3,4\}$ obtained as in Fig. 11 using the robust formulation with $\eta_{e}=0.4, \eta_{b}=0.5, \eta_{d}=0.6$. $\phi^{b}(\gamma=3)=-5.73$ and $\phi^{b}(\gamma=4)=-7.20$

be referred to some design elements not being totally $0-1$. When optimizing these two problems using the robust formulation, designs as shown in Fig. 14 appear. The small intermediate inclusions that caused the kinks seem to disappear and from the blue-print $\left(\eta_{b}=0.5\right)$ objective values $\phi^{b}(\gamma=3)=-5.73$ and $\phi^{b}(\gamma=4)=$ -7.20 it is clear, that the performance is slightly better than for the results obtained using the standard formulation $\phi(\gamma=3)=-5.68$ and $\phi(\gamma=4)=-6.90$ (see Fig. 11. Thus, simulating and using three realizations during optimization seems to be a feasible approach for suppressing the influence of intermediate design values if these appear to be a problem. It is important to note, that the computational effort is also three times larger. The simulation time can, however, be reduced to that of one simulation. As most workstations contain multiple cores and each design realization is independent from the others, parallelization is relatively straightforward. For the optimizer the process is slightly slower as the robust formulation introduce more constraints, which potentially slow down the convergence.

\subsection{Particle focusing device}

For the optimization of the particle-focusing device, two passive regions have been introduced to ensure a proper modeling in the inlet and outlet. The length of the inlet region is one channel height while the outlet region is three channel heights. This corresponds to the entrance length in a duct flow i.e. the length it takes to form a fully developed profile $L_{\text {entrance }} \approx 0.06 \mathrm{HRe}=$ $0.06 \frac{2 \rho u_{\max } H^{2}}{3 \mu}=3 H$ (Fox et al. 2004). Thus for designs obtained at $u_{\max }=75 \mathrm{~mm} / \mathrm{s}$ the flow should be close to fully developed at the outlet. We consider here three particles entering with velocity corresponding to the surrounding fluid. Recall the optimization goal is to confine the particle trajectories at the outlet of the flow domain. The design shown in Fig. 15(a) is obtained 


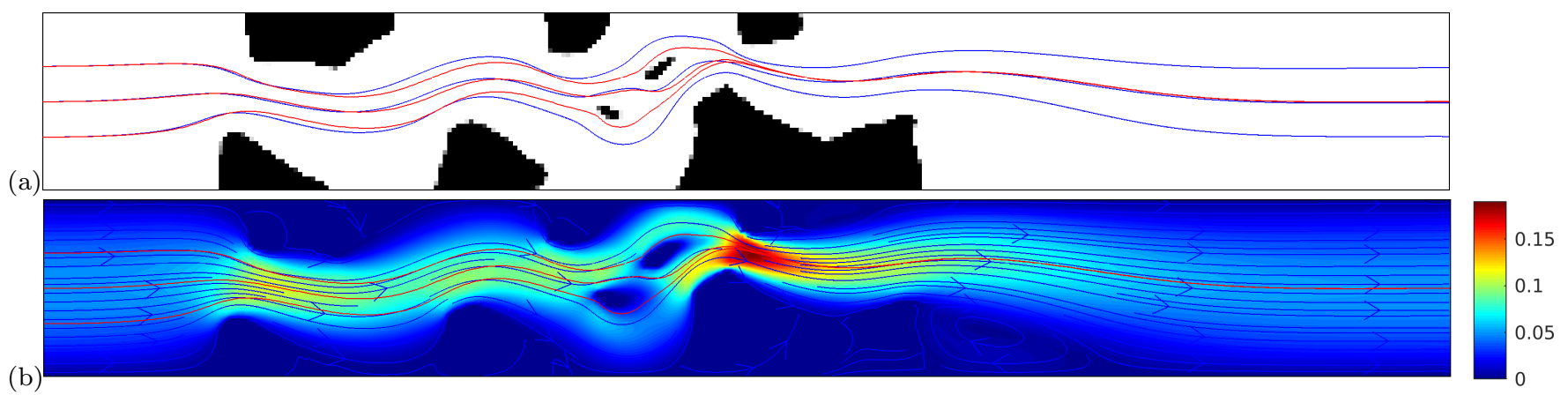

Fig. 15 Optimized design for particle focusing device $\left(R e=33 \frac{1}{3}, \theta=0.7, \gamma=10\right)$. Upper figure shows design with red particle trajectories and blue streamlines seeded in the particle release points. Lower figure shows velocity magnitude and streamlines.

for a maximum inlet velocity $u_{\max }=50 \mathrm{~mm} / \mathrm{s}$ under a pressure drop constraint of $\gamma=10$ and travel time constraints for each of the particles of $\theta=0.7$. The channel is indeed topology optimized and it is seen from the particle trajectories shown in red that they confine at $x_{2}=0.5 H$ near the outlet. The blue streamlines are seeded in the same points as the particles. They indicate where a neutral fluid particle would end up in the outlet. In $2 \mathrm{D}$, the motion is severely restricted such that the fluid cannot mix i.e. if both inlet and outlet are fully developed the streamline of any fluid particle would end in the same lateral position as it started. This of course also puts severe limitations on the outcome of any optimization.

Some isolated islands are observed in the main flow path. Since the present model assumes plane flow, the anchoring of such material should not be a problem.

Inspecting Fig. 15(b) showing the velocity magnitude it is clearly seen that the fluid velocity is penalized and close to zero in the solid regions. However, as also noticeable by the streamlines some fluid transport is taking place in these regions, which may be remedied by increasing the maximum impermeability further. Locally, the fluid velocity is increased, in some cases up to a factor of four, which increase the possibilities for the particles to migrate over streamlines.

The optimization history is shown in Fig. 16(a), which shows the error in particle confinement in a logarithmic scale to the left and the constraint functions on the right. It is seen from the kinks on the pressure drop constraint and the objective function that the continuation of the projection parameter and the corresponding restart of the optimization algorithm gives rise to some design adjustments. The final objective is $\phi=-13.17$ which is indeed a small confinement error.

Fig. 16(b) shows the velocity profiles for the inlet and outlet. It is clearly seen that the fully developed profile is very close to be obtained again at the outlet. The plot further show the lateral particle positions in
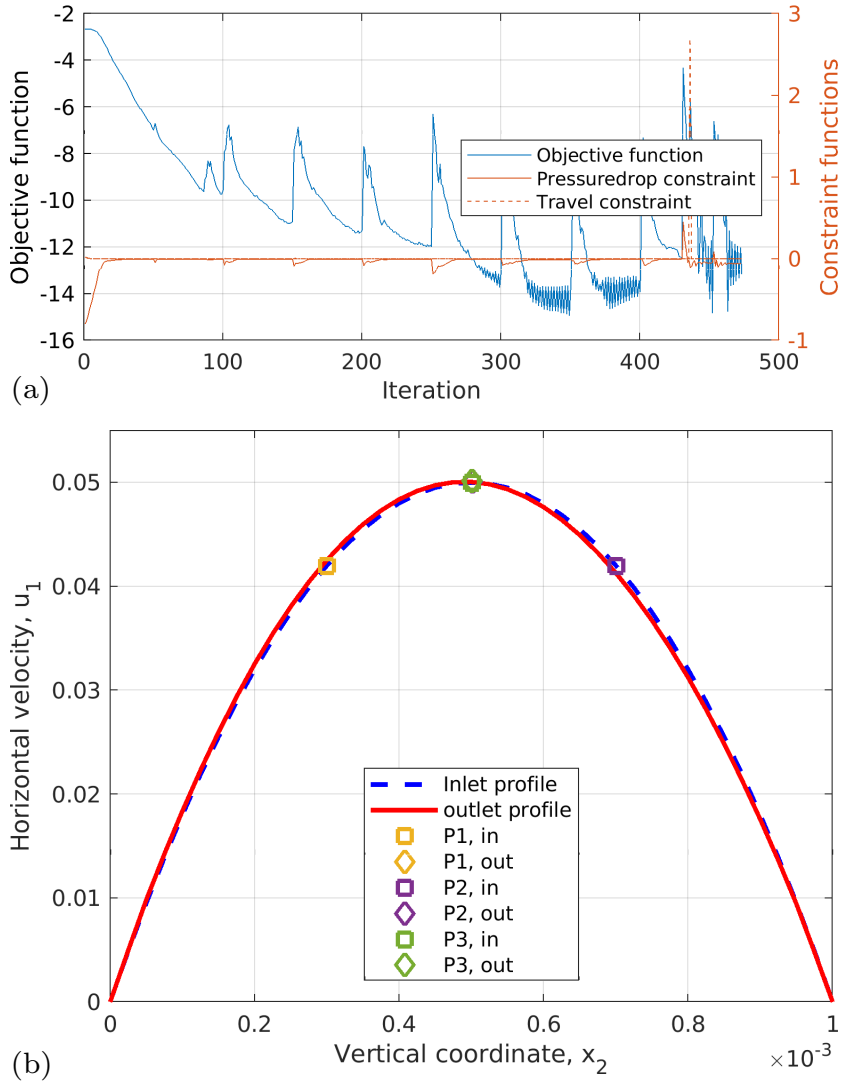

Fig. 16 (a) Objective and constraint history and (b) Velocity profile and particle position at inlet and outlet for the design in Fig. 15

the inlet and outlet and they have clearly confined in the desired position. Furthermore, it is seen that they exit the physical domain at the same speed as the fluid.

Fig. 17 shows the designs obtained for $R e=33 \frac{1}{3}$ with $\gamma=10$ allowing for a longer travel time $\theta=1$. Also the cases of decreased $\left(u_{\max }=25 \mathrm{~mm} / \mathrm{s} \Rightarrow R e=16 \frac{2}{3}\right)$ and increased $\left(u_{\max }=75 \mathrm{~mm} / \mathrm{s} \Rightarrow R e=50\right)$ inlet velocity for the former $\theta=0.7$ design are shown. The larger travel time clearly allows the particles to be obstructed more by the solid structure. It is clearly seen 
that the particles are traveling very close to the solid material, thus almost violating the assumption that the penalization of fluid velocity is sufficient to avoid particle flow in the solid. From the objective, this increased travel time does not improve the objective, which display a slightly decreased performance: $\phi=-12.11$.

For a decrease in the inlet velocity, we see that the optimizer is not able to confine the particle trajectories as well. This is also evident from the final objective $\phi=-10.08$ while the case of increased velocity there is so much freedom to the optimizer that the particles confine perfectly with a final objective $\phi=-13.28$. Thus, for larger flow rates a lower allowed pressure drop or more compact designs may be considered.

\subsection{Validation by post simulation}

The obtained design shown in Fig. 15 is extracted using an iso-contour in $\hat{\xi}=0.5$ and the design is exported to COMSOL for a post optimization study. A body-fitted triangular mesh with same maximum side length as for the elements used in the optimization procedure $\left(h_{e}=0.025 \mathrm{~mm}\right)$ is used in the simulation along with the same physical parameters. Using COMSOL's particle tracing module as done in Fig. 7 we obtain a qualitatively very good match shown in Fig. 18 . The red dots show the COMSOL particle trajectories while the cyan dots show the particle trajectories of Fig. 17. It is seen that there is some discrepancy in the final positions but a good overall correspondence. The exit particle positions in the COMSOL model are $x_{2}^{\text {post }}=\{0.492,0.509,0.549\} H$ while those of the optimization model were $x_{2}^{o p t}=\{0.500,0.500,0.501\} H$. The accurate modeling and actual location of the solid interface might differ slightly between the two models. This can be due to the choice of cut-off value for the iso-contour but also due to the existence of intermediate valued elements in the optimization model. Furthermore, the finite permeability in the optimization framework is in the COMSOL model interchanged with impermeable solid material as solid regions are excluded from the flow model. Inspecting Fig. 19 gives a hint about the reason for the loss of confinement in the postanalysis. From the magnification it can be seen that the trajectories for the two outermost particles, that the post analysis results are being pushed further apart laterally, most probably due to impermeable nature of the solid material in this model which force more fluid to pass around the solid instead of through it. This is also the case later along the upper trajectory where the top part of the nozzle forces the particle to accelerate more downwards than the optimization model leading to the loss of focus.

\subsection{Extensions to $3 \mathrm{D}$}

The designs presented are all $2 \mathrm{D}$ as the focus is on the development and demonstration of a framework for microfluidic particle manipulators. There are, however, much more to be gained by adding the third dimension. The possibility to exploit secondary motion as a means to control or manipulate the trajectory of the particles would have a significant influence on the performance. This is exemplified by e.g. Dean flow, where the secondary motion of a fluid flow in a helical tube is used for migrating particles (see e.g. Di Carlo et al. (2007)). In the mixing literature, it has been shown that chaotic advection can only appear in 3D c.f. Stroock (2002) and these effects may also apply for particle trajectories.

An extension to a 3D framework seems not too difficult building upon e.g. the TopOpt PETSc framework Aage et al. 2015, Alexandersen et al. 2016). The particle integrator used in the present work could potentially also be ported, however, one needs to pay attention to the specific needs and bottlenecks when doing parallel computing.

A more problematic issue might be that of solid islands in the flow path. In 3D the support structure is missing compared to $2 \mathrm{D}$ where islands can be assumed attached in the out-of-plane direction. One way to go about this would be to introduce a connectivity constraint based on a a virtual heat conduction problem with a virtual temperature field as done in e.g. Andreasen et al. (2014) for material design. The structure is loaded by a virtual design dependent heat source (in solid regions) and all boundaries are heat sinks. If solid is modeled as a good conductor and void as a poor, the connectivity can be ensured by limiting the maximum allowable virtual temperature. Other alternatives might be to restrict the design to be a $2 \mathrm{D}$ extruded design. Alternatively, it can be done in terms of imposing manufacturing constraints such as casting constrains (Gersborg and Andreasen 2011) or milling filters (Langelaar 2019) which both can ensure connectivity of the design, with the bonus of ensuring that the devices can actually be manufactured. However, this may overly restrict the design in cases where manufacturing of a complex structure is not a problem.

\section{Replication of results}

Many details have been given in the relevant sections however, for completeness the details / parameters used are listed in this section. 


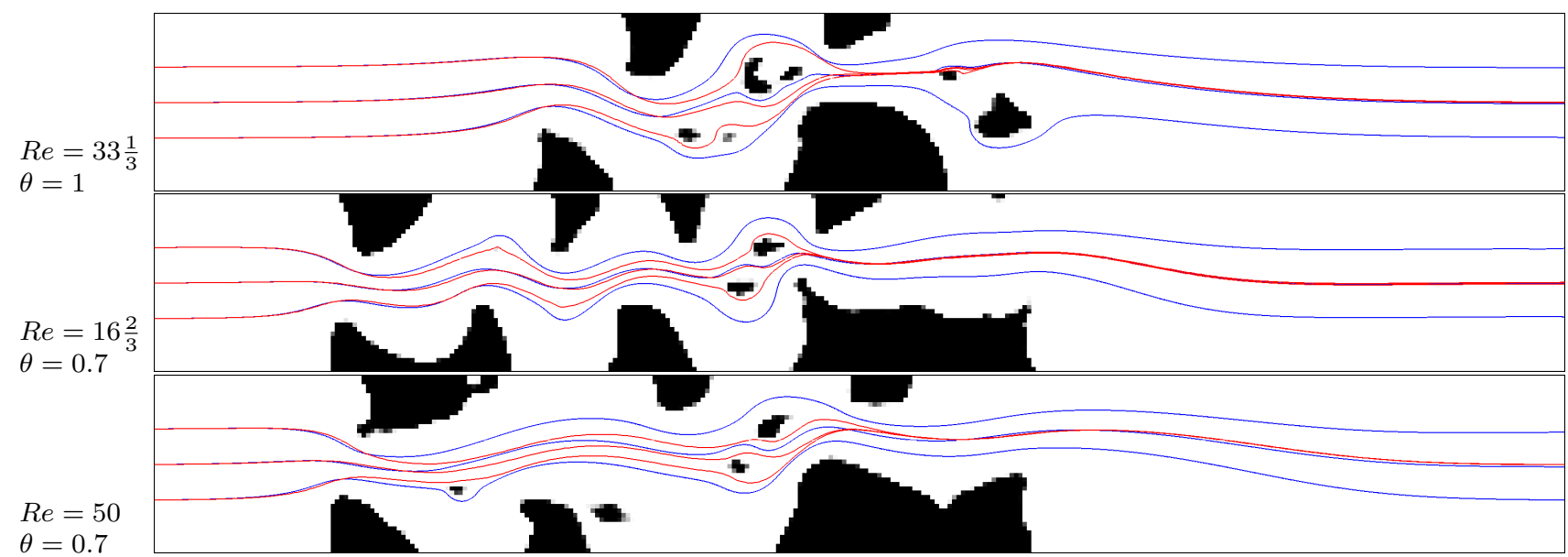

Fig. 17 Red lines are particle trajectories while blue lines are streamlines seeded at the same points. Upper: Optimized design for $\gamma=10, R e=33 \frac{1}{3}, \theta=1$. Center: Optimized design for $\gamma=10, R e=16 \frac{2}{3}, \theta=0.7$. Lower: Optimized design for $\gamma=10$, $R e=50, \theta=0.7$

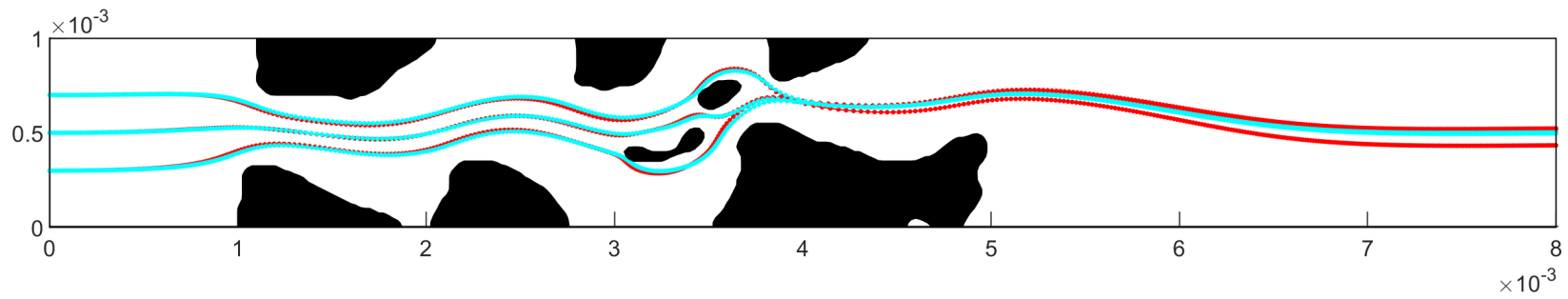

Fig. 18 Validation of the particle focusing device by remodeling in COMSOL using a body-fitted mesh for $u_{\max }=50 \mathrm{~mm} / \mathrm{s}$. Particle trajectory obtained for imported design in COMSOL shown in red. Trajectory from Fig. 15 in cyan.

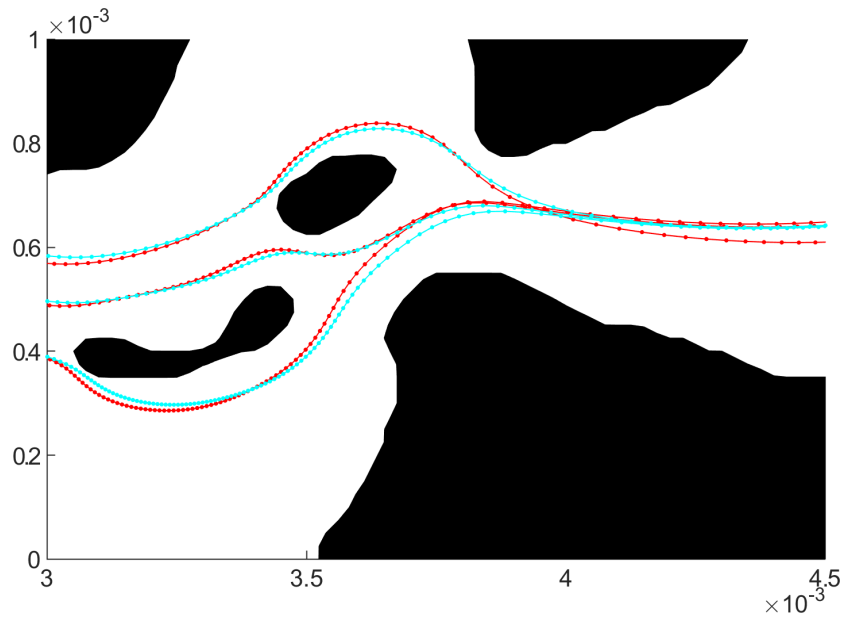

Fig. 19 Zoom of the center part of the post analysis result in 18

Filter / projection / continuation:

\begin{tabular}{ll}
\hline Projection threshold: & $\eta=0.5$ \\
Filter radius & $R=3$ elements
\end{tabular}

Projection steepness

$$
\beta=\{0.1,1.1,2.1,3.1,4,8,16,32,64\}
$$

Max inverse permeability: $\bar{\alpha}=10^{6}$

Min inverse permeability: $\quad \underline{\alpha}=0$

Penalization factor: $\quad q=1$
MMA/optimization parameters:

$\begin{array}{ll}\text { Asymptote initialization: } & 0.2 \\ \text { Asymptote decrease: } & 0.65 \\ \text { Asymptote increase: } & 1.05 \\ \text { External move limit: } & 0.1 \\ \text { Constraint penalization factor: } & \mathrm{c}=1000 \\ \text { Stopping criteria: } & \left\|\xi^{\text {new }}-\xi^{\text {old }}\right\|_{\infty}<1 \% \\ \text { Max iterations: } & 500\end{array}$

Optimization sequence only stopped if constraints are non-violated.

Time integrator (ode15s) settings:

\begin{tabular}{ll} 
Relative tolerance: & rtol $=10^{-8}$ \\
Absolute tolerance: & atol $=10^{-12}$ \\
Max step: & $\Delta t_{\max }=\frac{h_{e}}{2 u_{\max }}$ \\
\hline
\end{tabular}

Flow solver / problem:

$\begin{array}{ll}\text { Maximum iterations: } & 100 \\ \text { Relative tolerance: } & 10^{-8} \\ \text { Absolute tolerance: } & 10^{-14} \\ \text { Initial solution: } & \text { last solution }\end{array}$




\begin{tabular}{ll}
\multicolumn{2}{c}{ Trajectory problem: } \\
\hline Domain size: & $4 \mathrm{~mm} \times 1 \mathrm{~mm}$ \\
Mesh size: & $160 \times 40$ \\
Initial design: & $\xi_{\text {init }}=1$ (all fluid) \\
Particle seed point: & $x_{2}=0.7 \mathrm{H}$ \\
Particle density: & $\rho_{p}=1000 \mathrm{~kg} / \mathrm{m}^{3}$ \\
Particle diameter: & $d_{p}=10 \mu \mathrm{m}$ \\
Fluid viscosity: & $\mu=10^{-3} \mathrm{~Pa} . \mathrm{s}$ \\
Fluid density: & $\rho=1000 \mathrm{~kg} / \mathrm{m}^{3}$ \\
\hline & $\quad$ Focusing device: \\
\hline Domain size: & $8 \mathrm{~mm} \times 1 \mathrm{~mm}$ \\
Mesh size: & $320 \times 40$ \\
Initial design: & $\xi_{\text {init }}=1 \mathrm{except}$ solid \\
& half circle with center \\
& $\mathbf{x}=(L / 2,0)$ and radius $H / 2$. \\
Particles seed point: & $x_{1}=0$ \\
& $x_{2}=\{0.3 H, 0.5 H, 0.7 H\}$ \\
Particle density: & $\rho_{p}=8000 \mathrm{~kg} / \mathrm{m}^{3}$ \\
Particles diameter: & $d_{p}=40 \mu \mathrm{m}$ \\
Fluid viscosity: & $\mu=10^{-3} \mathrm{Pa.s}$ \\
Fluid density: & $\rho=1000 \mathrm{~kg} / \mathrm{m}^{3}$ \\
\hline
\end{tabular}

\section{Discussion and Conclusion}

The paper presents a framework to optimize microfluidic components with particle control abilities. The work should be seen as a demonstrator of the potential that lie in design synthesis by topology optimization of particle control devices. The mobility in $2 \mathrm{D}$ is rather limited and an extension to 3D would definitely allow for much more particle movement as e.g. Dean forcing (secondary motion in a curved channel) could be utilized. The methodology includes a Heaviside projection filtering in order to clean the designs, as there is only limited intrinsic penalization in the problem formulation. The design influence of the given constraining parameters, pressure drop and travel time, have been investigated for the particle trajectory problem while the effect of Reynolds number have been the focus of the particle focusing design.

The design optimization method chosen here is topology optimization, which may be discussed. Is topology optimization necessary for flow optimization? For simple channels where pressure minimization is the main concern, the answer might be no, however, in a case like particle control, the ability to synthesize a complex geometry with multiple solid (non-fluid) parts is needed indeed. If a shape optimization procedure should be used instead, it should be at least one in which large boundary alterations are supported.

For the presented problems the assumption of not modeling the interaction with the solid material seem to hold, however, this could potentially be problematic if the fluid containing the particles was a gas instead of a liquid yielding a much higher contrast in mass density. In this respect, other assumptions might also be violated. The current work does not include any diffusion i.e. there is no Brownian forcing of the particles and the fluid is assumed laminar. However, these are definitely applicable extensions but they may very well make the computation time grow dramatically. Similarly, the restrictions on the suspension being dilute and one-way coupled to the fluid flow may also be worked upon by adding particle-particle interaction and a fully coupled particle-fluid solver. The design of the particle-focusing device was post analyzed a commercial software, COMSOL 5.4, using a body-fitted mesh. The focusing ability was maintained, however, some of the fine-tuning of focusing was lost either in the conversion from one design representation to another or due to the switch from modeling the solid using an immersed method to using a segregated model.

Acknowledgements The author would like to thank the audience at WCSMO13 for constructive comments on the part of the work presented there. Furthermore, M.Sc. Jeppe Alexander Christensen, Associate Professor Niels Aage and the TopOpt group at the Technical University of Denmark is acknowledged for fruitful discussions on the work.

\section{Conflict of interest}

The author states that there is no conflict of interest.

\section{References}

Aage N, Andreassen E, Lazarov BS (2015) Topology optimization using PETSc: An easy-to-use, fully parallel, open source topology optimization framework. Structural and Multidisciplinary Optimization 51(3):565-572, DOI 10.1007/s00158-014-1157-0

Alexandersen J, Aage N, Andreasen CS, Sigmund O (2014) Topology optimisation for natural convection problems. International Journal for Numerical Methods in Fluids 76(10):699-721, DOI 10.1002/fld.3954

Alexandersen J, Sigmund O, Aage N (2016) Large scale three-dimensional topology optimisation of heat sinks cooled by natural convection. International Journal of Heat and Mass Transfer 100:876-891, DOI 10.1016/j. ijheatmasstransfer.2016.05.013

Andreasen CS (2017) Topology optimization of inertia driven dosing units. Structural and Multidisciplinary Optimization 55(4):1301-1309, DOI 10.1007/s00158-016-1573-4

Andreasen CS, Gersborg AR, Sigmund O (2009) Topology optimization of microfluidic mixers. International Journal for Numerical Methods in Fluids 61(5):498-513, DOI 10. 1002/fld.1964

Andreasen CS, Andreassen E, Jensen JS, Sigmund O (2014) On the realization of the bulk modulus bounds for twophase viscoelastic composites. Journal of the Mechanics 
and Physics of Solids 63(1):228-241, DOI 10.1016/j.jmps. 2013.09.007

Behrou R, Ranjan R, Guest JK (2019) Adaptive topology optimization for incompressible laminar flow problems with mass flow constraints. Computer Methods in Applied Mechanics and Engineering 346:612-641, DOI 10.1016/j.cma.2018.11.037

Bendsøe MP, Kikuchi N (1988) Generating optimal topologies in structural design using a homogenization method. Computer Methods in Applied Mechanics and Engineering 71(2):197-224, DOI 10.1016/0045-7825(88)90086-2

Borrvall T, Petersson J (2003) Topology optimization of fluids in Stokes flow. International Journal for Numerical Methods in Fluids 41(1):77-107, DOI 10.1002/fld.426

Chan PCH, Leal LG (1979) The motion of a deformable drop in a second-order fluid. Journal of Fluid Mechanics 92(1):131-170, DOI 10.1017/S0022112079000562

Deng Y, Liu Z, Zhang P, Wu Y, Korvink JG (2010) Optimization of no-moving part fluidic resistance microvalves with low reynolds number. In: 2010 IEEE 23rd International Conference on Micro Electro Mechanical Systems (MEMS), IEEE, pp 67-70, DOI 10.1109/MEMSYS.2010. 5442565

Deng Y, Liu Z, Zhang P, Liu Y, Wu Y (2011) Topology optimization of unsteady incompressible Navier-Stokes flows. Journal of Computational Physics 230(17):66886708, DOI 10.1016/j.jcp.2011.05.004

Di Carlo D, Irimia D, Tompkins RG, Toner M (2007) Continuous inertial focusing, ordering, and separation of particles in microchannels. Proceedings of the National Academy of Sciences of the United States of America 104(48):18892-18897, DOI 10.1073/pnas.0704958104

Dilgen CB, Dilgen SB, Fuhrman DR, Sigmund O, Lazarov BS (2018a) Topology optimization of turbulent flows. Computer Methods in Applied Mechanics and Engineering 331:363-393, DOI 10.1016/j.cma.2017.11.029

Dilgen SB, Dilgen CB, Fuhrman DR, Sigmund O, Lazarov BS (2018b) Density based topology optimization of turbulent flow heat transfer systems. Structural and Multidisciplinary Optimization 57(5):1905-1918, DOI 10.1007/ s00158-018-1967-6

Fox RW, McDonald AT, Pritchard PJ (2004) Introduction to Fluid Mechanics, 6th edn. John Wiley \& Sons

Gale B, Jafek A, Lambert C, Goenner B, Moghimifam H, Nze U, Kamarapu S (2018) A Review of Current Methods in Microfluidic Device Fabrication and Future Commercialization Prospects. Inventions 3(3):60, DOI 10.3390/ inventions 3030060

Gersborg AR, Andreasen CS (2011) An explicit parameterization for casting constraints in gradient driven topology optimization. Structural and Multidisciplinary Optimization 44(6):875-881, DOI 10.1007/s00158-011-0632-0

Gersborg-Hansen A, Sigmund O, Haber R (2005) Topology optimization of channel flow problems. Structural and Multidisciplinary Optimization 30(3):181-192, DOI 10.1007/s00158-004-0508-7

Guest JK, Prévost JH (2006) Topology optimization of creeping fluid flows using a Darcy-Stokes finite element. International Journal for Numerical Methods in Engineering 66(3):461-484, DOI 10.1002/nme.1560

Guo Y, Xu Y, Deng Y, Liu Z (2018) Topology Optimization of Passive Micromixers Based on Lagrangian Mapping Method. Micromachines 9(3):137, DOI 10.3390/ mi9030137

Hyun Jc, Hyun J, Wang S, Yang S (2017) Improved pillar shape for deterministic lateral displacement separa- tion method to maintain separation efficiency over a long period of time. Separation and Purification Technology 172:258-267, DOI 10.1016/j.seppur.2016.08.023

Kang BS, Park GJ, Arora JS (2006) A review of optimization of structures subjected to transient loads. Structural and Multidisciplinary Optimization 31(2):81-95, DOI 10.1007/s00158-005-0575-4

Kim D, Kim Y, Lee D, Kim B, Lee J (2017) Electrode configuration optimization for maximizing throughput of dielectrophoretic particle separator. Journal of Mechanical Science and Technology 31(12):5951-5960, DOI 10.1007/s12206-017-1139-4

Kreissl S, Maute K (2012) Levelset based fluid topology optimization using the extended finite element method. Structural and Multidisciplinary Optimization 46(3):311-326, DOI 10.1007/s00158-012-0782-8

Kreissl S, Pingen G, Evgrafov A, Maute K (2010) Topology optimization of flexible micro-fluidic devices. Structural and Multidisciplinary Optimization 42(4):495-516, DOI 10.1007/s00158-010-0526-6

Kreissl S, Pingen G, Maute K (2011) An explicit level set approach for generalized shape optimization of fluids with the lattice Boltzmann method. International Journal for Numerical Methods in Fluids 65(5):496-519, DOI 10.1002/fld.2193

Lalli F, Esposito PG, Piscopia R, Verzicco R (2005) Fluid-particle flow simulation by averaged continuous model. Computers \& Fluids 34(9):1040-1061, DOI 10. 1016/j.compfluid.2004.08.004

Langelaar M (2019) Topology optimization for multi-axis machining. Computer Methods in Applied Mechanics and Engineering 351:226-252, DOI 10.1016/j.cma.2019.03.037

Manninen M, Taivassalo V, Kallio S, Akademi Å (1996) On the mixture model for multiphase flow, vtt public edn. VTT

Marck G, Nemer M, Harion JL (2013) Topology Optimization of Heat and Mass Transfer Problems: Laminar Flow. Numerical Heat Transfer, Part B: Fundamentals 63(6):508539, DOI 10.1080/10407790.2013.772001

Maxey MR, Riley JJ (1983) Equation of motion for a small rigid sphere in a nonuniform flow. Physics of Fluids 26(4):883-889, DOI 10.1063/1.864230

Michaleris P, Tortorelli DA, Vidal CA (1994) Tangent operators and design sensitivity formulations for transient non-linear coupled problems with applications to elastoplasticity. Int J Numer Meth Engng 37(14):2471-2499, DOI 10.1002/nme.1620371408

Myung JH, Hong S (2015) Microfluidic devices to enrich and isolate circulating tumor cells. Lab on a Chip 15(24):4500-4511, DOI 10.1039/C5LC00947B

Okkels F, Bruus H (2007) Scaling behavior of optimally structured catalytic microfluidic reactors. Physical Review E 75(1):016301, DOI 10.1103/PhysRevE.75.016301

Olhoff N (1989) Multicriterion structural optimization via bound formulation and mathematical programming. Structural Optimization 1(1):11-17, DOI 10.1007/ BF01743805

Özbey A, Karimzadehkhouei M, Akgönül S, Gozuacik D, Koşar A (2016) Inertial focusing of microparticles in curvilinear microchannels. Scientific Reports 6(1):38809, DOI 10.1038/srep38809

Park J, Kim B, Choi SK, Hong S, Lee SH, Lee KI (2005) An efficient cell separation system using 3D-asymmetric microelectrodes. Lab on a Chip 5(11):1264, DOI 10.1039/ b506803g 
Schott B, Ager C, Wall WA (2019) Monolithic cut finite element-based approaches for fluid-structure interaction. International Journal for Numerical Methods in Engineering 119(8):757-796, DOI 10.1002/nme.6072

Shampine LF, Reichelt MW (1997) The MATLAB ode suite. SIAM Journal of Scientific Computing 18(1):1-22, DOI 10.1137/S1064827594276424

Stan CA, Ellerbee AK, Guglielmini L, Stone HA, Whitesides GM (2013) The magnitude of lift forces acting on drops and bubbles in liquids flowing inside microchannels. Lab Chip 13(3):365-376, DOI 10.1039/C2LC41035D

Stroock AD (2002) Chaotic Mixer for Microchannels. Science 295(5555):647-651, DOI 10.1126/science.1066238

Svanberg K (1987) The Method Of Moving Asymptotes - A New Method For Structural Optimization. International Journal for Numerical Methods in Engineering 24(2):359373

Tezduyar TE, Ramakrishnan S, Sathe S (2008) Stabilized formulations for incompressible flows with thermal coupling. International Journal for Numerical Methods in Fluids 57(9):1189-1209, DOI 10.1002/fld.1743

Villanueva CH, Maute K (2017) CutFEM topology optimization of 3D laminar incompressible flow problems. Computer Methods in Applied Mechanics and Engineering 320:444-473, DOI 10.1016/j.cma.2017.03.007

Wang F, Jensen JS, Sigmund O (2011) Robust topology optimization of photonic crystal waveguides with tailored dispersion properties. Journal of the Optical Society of America B 28(3):387, DOI 10.1364/JOSAB.28.000387

White FM (1991) Viscous Fluid Flow, 2nd edn. McGraw-Hill Book Co.

Yoon GH (2010) Topology optimization for stationary fluidstructure interaction problems using a new monolithic formulation. International Journal for Numerical Methods in Engineering 82(5):591-616, DOI 10.1002/nme.2777

Yoon GH, Park J (2010) Topological design of electrode shapes for dielectrophoresis based devices. Journal of Electrostatics 68(6):475-486, DOI 10.1016/J.ELSTAT. 2010.06.008 